\title{
Organization of Disparity-Selective Neurons in Macaque Area MT
}

\author{
Gregory C. DeAngelis and William T. Newsome \\ Howard Hughes Medical Institute and Department of Neurobiology, Stanford University School of Medicine, \\ Stanford, California 94305-5401
}

Neurons selective for binocular disparity are found in a number of visual cortical areas in primates, but there is little evidence that any of these areas are specialized for disparity processing. We have examined the organization of disparity-selective neurons in the middle temporal visual area (MT), an area shown previously to contain an abundance of disparity-sensitive neurons. We recorded extracellularly from MT neurons at regularly spaced intervals along electrode penetrations that passed through MT either normal to the cortical surface or at a shallow oblique angle. Comparison of multiunit and single-unit recordings shows that neurons are clustered in MT according to their disparity selectivity. Across the surface of MT, disparityselective neurons are found in discrete patches that are separated by regions of $\mathrm{MT}$ that exhibit poor disparity tuning. Within disparity-selective patches of MT, we typically observe a

A common feature of cortical organization is the clustering of neurons with similar functional properties into columns that traverse the cortical sheet. Since Mountcastle (1957) first discovered columnar organization in the somatosensory cortex, it has been observed in many regions of the neocortex, including several visual, auditory, and somatosensory areas (for review, see Mountcastle, 1997). In the visual system, Hubel and Wiesel (1977) demonstrated columns for contour orientation and ocular dominance in striate cortex (V1). More recently, columnar organization in V1 has been described for other visual parameters, including spatial frequency (e.g., Tootell et al., 1981; Tolhurst and Thompson, 1982; Shoham et al., 1997) and direction of motion (e.g., Payne et al., 1980; Shmuel and Grinvald, 1996; Weliky et al., 1996). Columns exist in many extrastriate visual areas as well. The middle temporal visual area (MT), for example, contains columns for direction of motion (Albright et al., 1984), area V4 is reported to exhibit columns for wavelength (Zeki, 1973), and inferotemporal cortex is thought to contain columns for complex visual features, including faces (Fujita et al., 1992).

Binocular disparity has received relatively little attention with regard to functional organization. Neurons selective for binocular disparity were first described in cat striate cortex three decades ago (Barlow et al., 1967; Pettigrew et al., 1968), and disparity-

Received July 7, 1998; revised Oct. 20, 1998; accepted Dec. 2, 1998.

G.C.D. was supported by a Medical Research Fellowship from the Bank of America/Giannini Foundation, by a National Research Service Award from the National Eye Institute, and by a Career Award in the Biomedical Sciences from the Burroughs-Wellcome Fund. W.T.N. is an investigator of the Howard Hughes Medical Institute. This work was also supported by National Eye Institute Grant EY05603. We are grateful to Judy Stein for assistance with histology and to Bruce Cumming, Jamie Nichols, and Jing Liu for helpful comments on this manuscript.

Correspondence should be addressed to Dr. William T. Newsome, Department of Neurobiology, Stanford University School of Medicine, Stanford, CA 94305-5401.

Copyright (C) 1999 Society for Neuroscience $0270-6474 / 99 / 191398-18 \$ 05.00 / 0$ smooth progression of preferred disparities (e.g., near to far) as our electrode travels parallel to the cortical surface. In electrode penetrations normal to the cortical surface, on the other hand, MT neurons generally have similar disparity tuning, with little variation from one recording site to the next. Thus disparitytuned neurons are organized into cortical columns by preferred disparity, and preferred disparity is mapped systematically within larger, disparity-tuned patches of MT. Combined with other recent findings, the data suggest that MT plays an important role in stereoscopic depth perception in addition to its well known role in motion perception.

Key words: visual cortex; binocular disparity; stereopsis; extrastriate; columnar organization; middle temporal; functional architecture selective cells have since been observed in several primate visual areas, including $\mathrm{V} 1, \mathrm{~V} 2, \mathrm{~V} 3, \mathrm{MT}$, and the medical superior temporal area (MST) (Hubel and Wiesel, 1970; Poggio and Fischer, 1977; Maunsell and Van Essen, 1983b; Burkhalter and Van Essen, 1986; Felleman and Van Essen, 1987; Hubel and Livingstone, 1987; Poggio et al., 1988; Roy et al., 1992). Despite this abundance of disparity-selective neurons, definitive evidence of a columnar organization of disparity has been lacking. Blakemore (1970) originally proposed the existence of constant-disparity columns in cat V1, but LeVay and Voigt (1988), using quantitative techniques, demonstrated only a weak clustering of neurons by preferred disparity. In primate V2, disparity-selective neurons occur predominantly in the thick cytochrome oxidase stripes and are reported to be clustered by disparity preference (i.e., near, zero, and far) within these regions (Hubel and Livingstone, 1987; Ts'o et al., 1990; Peterhans and von der Heydt, 1993; Roe and Ts'o, 1995). These reports suggest that binocular disparity is mapped systematically within the thick stripes of V2, although the data were not analyzed quantitatively. Disparity columns have also been reported in V2 of the sheep (Clarke et al., 1976).

We have examined the organization of binocular disparity in extrastriate visual area MT, which is well known for its role in motion perception (e.g., Zeki, 1978a; Albright, 1993). Maunsell and Van Essen (1983b) reported 16 years ago that two-thirds of MT neurons are highly selective for binocular disparity. Consistent with this observation, MT receives ascending input from the thick stripes of V2, suggesting that MT might inherit some organization for disparity from its inputs (DeYoe and Van Essen, 1985; Shipp and Zeki, 1985, 1989). Thus, we sought to determine whether MT neurons are organized in columns by disparity preference, as they are by direction preference (Albright et al., 1984). 


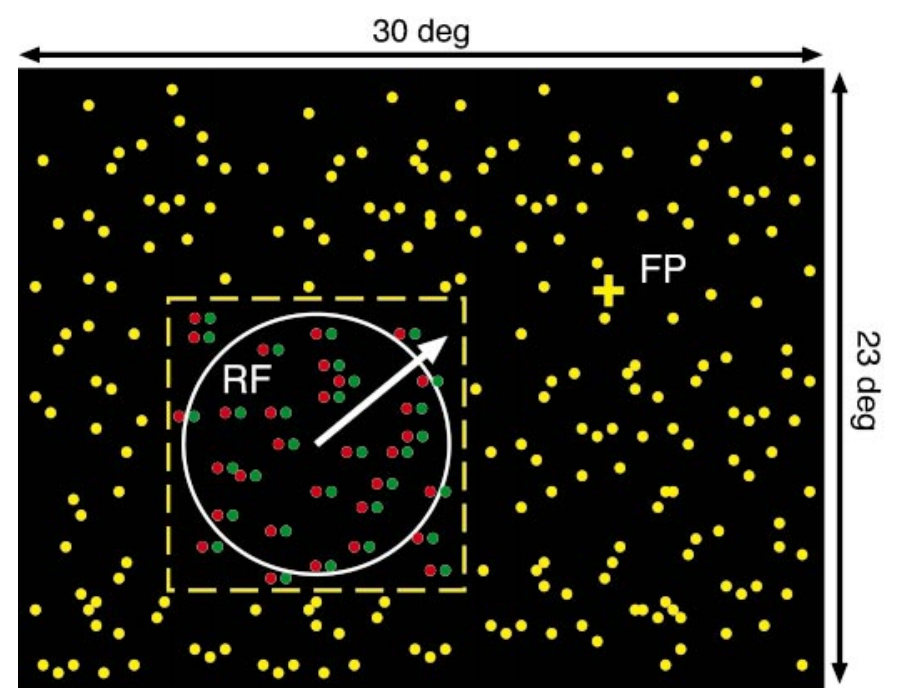

Figure 1. Schematic illustration of the visual stimulus. The fixation point $(F P)$ is indicated by a yellow cross. Moving dots of variable disparity were presented within a square aperture (dashed yellow lines) that was centered over and slightly larger than the receptive field $(R F$, white circle) of the recorded MT neurons. The white arrow denotes the direction of motion of the dots. Dots rendered outside the plane of fixation are shown as horizontally disparate red and green pairs, in which the red dots indicate the left eye's image and the green dots denote the right eye's image. Yellow dots outside the square aperture were presented at zero disparity and provided a background.

Our results reveal an elaborate functional organization for disparity in MT. Disparity-selective neurons are organized in a patchy manner, with some regions of MT exhibiting strong disparity selectivity and others having weak selectivity. Within regions of strong disparity tuning, moreover, the preferred disparity changes systematically across the surface of MT, while remaining fairly constant within vertical penetrations. These results reinforce the notion that MT is an important area for the analysis of disparity signals (Maunsell and Van Essen, 1983b; Bradley et al., 1995); disparity information carried by MT neurons may contribute to a number of perceptual capacities, including stereopsis. In agreement with this idea, we have reported recently that perceptual judgments of stereoscopic depth can be influenced in a predictable manner by electrical microstimulation of disparity columns in MT (DeAngelis et al., 1998).

\section{MATERIALS AND METHODS}

Our methods for surgical preparation, training, and electrophysiological recording from rhesus monkeys (Macaca mulatta) are described in detail elsewhere (Britten et al., 1992). Here we briefly describe our methods, focusing mainly on aspects that are most relevant to this study. Extracellular recordings were made from three hemispheres in two adult monkeys, one male and one female. Each animal was implanted with a stainless-steel head post and a scleral search coil for monitoring eye movements (Robinson, 1963; Judge et al., 1980). For a subset of experiments, monkey S had eye coils implanted in both eyes to monitor the vergence state of the eyes. During recording sessions, each monkey was seated in a primate chair with its head restrained via the head post. Behavioral training was accomplished using standard operant conditioning procedures; desired behavior on each trial was rewarded with a small amount of water or juice. All animal care and experimental procedures conformed to guidelines established by the National Institutes of Health.

Visual stimuli and task. Figure 1 illustrates the visual display that monkeys viewed in these experiments. On each trial, a small yellow fixation cross first appeared on a black background, and the monkey was required to maintain fixation within a $3^{\circ} \times 3^{\circ}$ electronic window centered on the cross. Trials in which the monkey broke fixation before the end of the visual stimulus were aborted immediately. Data were discarded on these trials, and the monkey was not rewarded.

After the monkey fixated the central cross, a bipartite random-dot pattern appeared for $1.5 \mathrm{sec}$. This pattern contained a central square aperture, centered on and slightly larger than the receptive field (RF), in which dots moved coherently in a direction of motion that could be varied systematically (e.g., Fig. 1, white arrow). When dots reached the edge of the aperture, they "wrapped around" and continued their trajectory from the opposite side of the aperture. In addition, this square patch of dots could be rendered in depth by presenting it as a red and green anaglyph that the monkey viewed through red and green filters (Kodak Wratten 29 and 61, respectively). Outside this square aperture, the remainder of the visual display was filled with zero-disparity (yellow) dots that were randomly replotted at $20 \mathrm{~Hz}$ to produce a twinkling background. This background helped anchor the monkey's vergence at the depth of the fixation point (see Discussion). Each dot had a size of $\sim 0.1^{\circ}$, and the dot pattern had a density of $32 \mathrm{dots} \cdot \mathrm{deg}^{-2} \cdot \mathrm{sec}^{-1}$.

The visual stimulus was presented on a standard 17 inch color display (Nanao T2-17), which subtended $30^{\circ} \times 23^{\circ}$ at the viewing distance of 57 $\mathrm{cm}$. The display was refreshed at $60 \mathrm{~Hz}$. The red and green guns of the display were calibrated through the red and green filters to match the luminances seen by the two eyes. The resulting effective luminance was $7.7 \mathrm{~cd} / \mathrm{m}^{2}$ when red dots were viewed through the red filter and green dots were viewed through the green filter. Viewed through the opposite filters, green dots had a luminance of $0.4 \mathrm{~cd} / \mathrm{m}^{2}$, and red dots had a luminance of $0.33 \mathrm{~cd} / \mathrm{m}^{2}$. Thus, the filters achieved reasonable stereo separation, and "ghosting" artifacts (faint shadows that appear at zero disparity) were barely visible.

Recording cylinder placement and electrode penetration angles. Recording cylinders were implanted in two different locations to allow access to MT from angles oblique and approximately normal to the cortical surface, as illustrated schematically in Figure $2 A$. A recording cylinder was implanted over the occipital lobe of both monkeys. In penetrations from this cylinder, electrodes were angled $20^{\circ}$ below the horizontal and traveled toward MT in a sagittal plane, passing first through the lunate sulcus (Fig. 2A). Thus, electrodes passed through MT at an oblique angle, ranging from 45 to $90^{\circ}$ away from the surface normal. We refer to these as "oblique" penetrations.

To make electrode penetrations approximately normal to the surface of MT, we implanted a recording cylinder over the frontal cortex in monkey P. Penetrations made through this cylinder were in a nearsagittal plane, with the electrodes angled $45^{\circ}$ below the horizontal. Thus, electrodes passed first through the central and intraparietal sulci before crossing the superior temporal sulcus and entering MT approximately normal to the cortical surface (Fig. $2 A$ ). We placed marking pins in the brain of this animal before perfusion so that we could accurately reconstruct the trajectories of "normal" penetrations made through the frontally placed cylinder. Figure $2 B$ shows a Nissl-stained section from this hemisphere that shows the trajectories of two of the marking pins as they passed through the superior temporal sulcus from dorsoanterior to ventroposterior. Faint traces of old microelectrode tracks are also visible in the white matter dorsal to MST on the anterior bank of the sulcus and ventral to MT on the posterior bank (Fig. $2 B$, arrows). The two marking pins bracketed the dorsoventral extent of all normal microelectrode penetrations in our study. Because of the curvature of the sulcus in the sampled region of cortex, the two marking pins provide an estimate of the maximal departure from normality of our microelectrode penetrations (assuming that the electrodes traveled along a straight path; if not, the deviations from normality could have been larger). The dorsal marking pin passed through MT at an angle $20^{\circ}$ from the normal, whereas the ventral marking pin passed through at an angle $-13^{\circ}$ from the normal. Clearly, microelectrode penetrations falling between these two marking pins were closer to the normal.

Data collection. Tungsten microelectrodes (impedance typically of 0.5-1.0 M $\Omega$; MicroProbe) were inserted into visual cortex through a transdural guide tube. Neural voltage signals were amplified and discriminated using conventional electronic equipment (Bak Electronics, Rockville, MD), and all event times were stored on a magnetic disk with 1 msec resolution. A multiunit (MU) event was defined as any deflection of the analog voltage signal that exceeded a threshold level set using a bilevel window discriminator (the waveform had to pass above one voltage-time cursor and below another). Therefore, an increase in the amplitude of the neural "hash" was transduced into an increase in the frequency of the MU response, which we express in events per second. The absolute frequency of the MU response is somewhat arbitrary, 


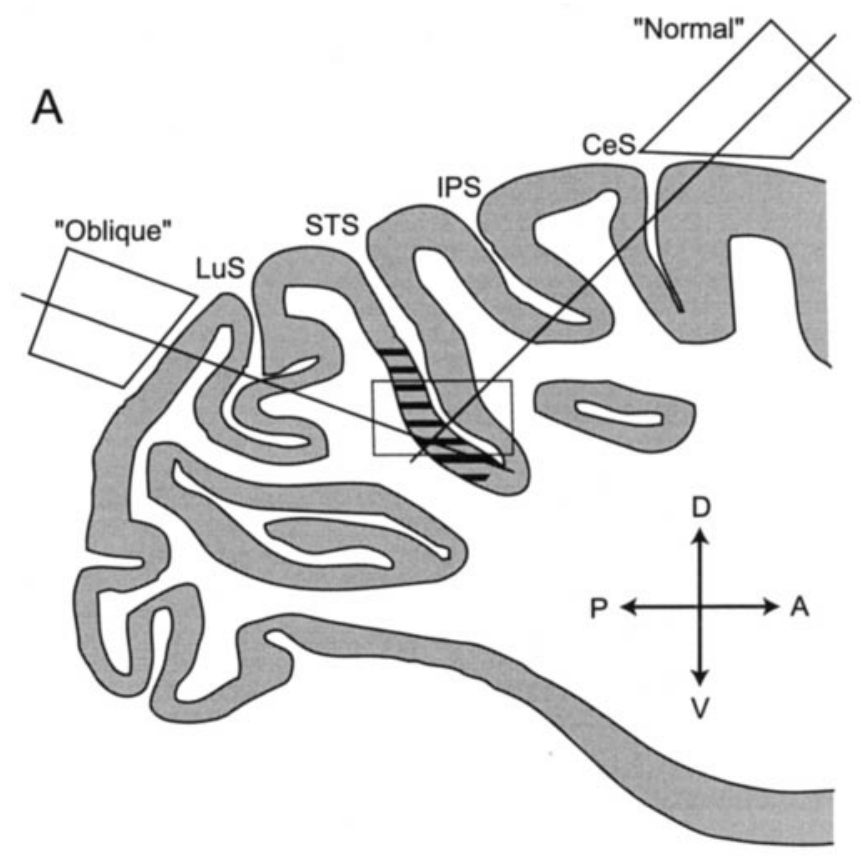

B

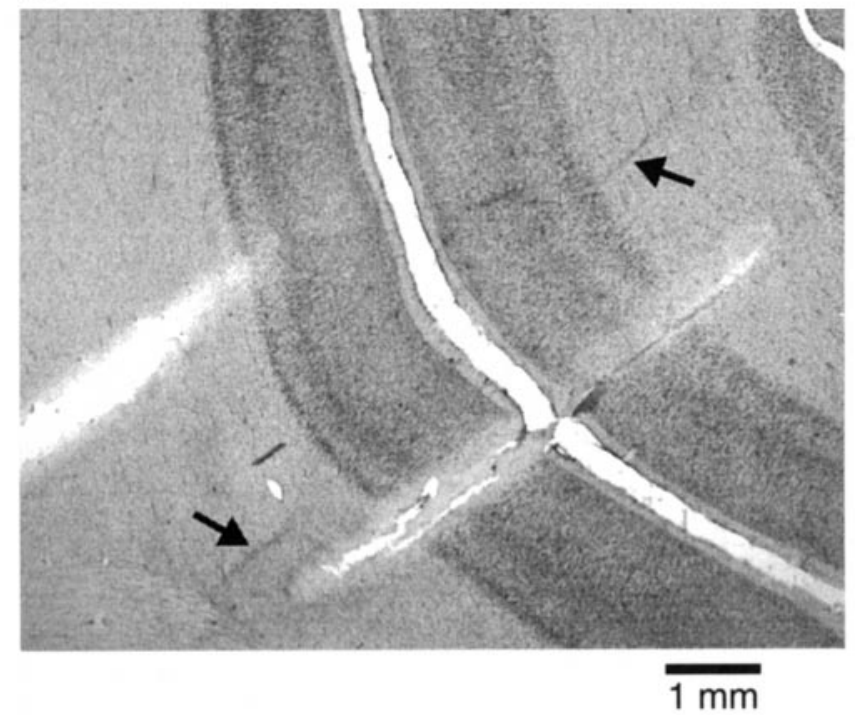

Figure 2. Illustration of electrode penetrations through MT. A, Schematic diagram showing the locations of recording cylinders and the electrode approaches to MT. The drawing is of a sagittal section through the brain of a monkey not used in the present study; shaded regions indicate gray matter. The hatched region of gray matter represents MT. Lines emerging from the recording cylinders (Normal and Oblique) indicate typical electrode trajectories from the two recording cylinders. For oblique penetrations, the recording cylinder was mounted over the occipital lobe at an angle $20^{\circ}$ below the horizontal. For normal penetrations, the recording cylinder was mounted over the precentral gyrus at an angle $45^{\circ}$ below the horizontal. $A$, Anterior; $C e S$, central sulcus; $D$, dorsal; IPS, intraparietal sulcus; $L u S$, lunate sulcus; $P$, posterior; $S T S$, superior temporal sulcus; and $V$, ventral. $B$, Nissl-stained section from the right hemisphere of monkey $\mathrm{P}$, which was implanted with the frontally located cylinder. The section depicts a midportion of the superior temporal sulcus, approximately equivalent to the rectangular box in $A$, in a nearsagittal plane. The two large scars were made by marking pins that were implanted just before perfusion along the trajectory of the normal microelectrode penetrations. Faint scars from microelectrode penetrations are also visible (arrows). Scale bar, $1 \mathrm{~mm}$. depending on the level of the event threshold as well as on the frequency bandpass of the amplifier. We reduced the arbitrary nature of this measurement by fixing the filter bandpass on our amplifier $(0.5-5 \mathrm{kHz})$ and by adjusting the event threshold at each recording site to obtain a spontaneous activity level in the range from 50 to 150 events/sec [mean, $98.7 \pm 34.7$ events/sec $( \pm \mathrm{SD}) ; n=411$ ]

Whenever possible, we recorded the times of occurrence of action potentials from an isolated single unit (SU) in addition to the MU response. SU isolation was achieved by feeding a separate copy of the voltage signal into a template-based spike discriminator (Alpha-Omega). We attempted to exclude SU spikes from the MU response by setting the upper level of the MU window discriminator below the peak of the SU spike waveform. To ensure that leakage of SU spikes into the MU record did not compromise our results, we conducted post hoc pruning for several data sets, removing all events from the MU record that occurred within $\pm 2 \mathrm{msec}$ of an SU spike. This pruning lowered the MU response by a few percent but had an otherwise negligible effect.

The practice of excluding SU spikes from the MU record (using the window discriminator) creates a series of "dead times" in the MU response, which generally reduced the measured MU response by a few percent when the SU was firing strongly (e.g., an SU firing at 50 spikes/sec will reduce the measured MU response by at most $5 \%$, because the sampling frequency is $1000 \mathrm{~Hz}$ ). Because the peak response rate of SUs was typically more than an order of magnitude weaker than that of the MU response (see Results), however, these dead times had a minimal effect on the MU tuning curves.

Horizontal and vertical eye position signals were sampled and stored to disk at $250 \mathrm{~Hz}$.

Experimental protocol and data analysis. Measurements were made at regularly spaced intervals of either 100 or $150 \mu \mathrm{m}$ along oblique and normal penetrations through MT. At each recording site, we first used an interactive computer "search" program to estimate RF location and size, preferred direction and speed of motion, and the range of disparities signaled by the neuron. These estimates were then used as the starting point for subsequent quantitative tests.

Figure 3 shows the sequence of quantitative measurements that we made at each recording site. We first measured a direction-tuning curve by presenting eight directions of motion, $45^{\circ}$ apart; each stimulus was presented four times. Figure $3 A$ illustrates an example. The data were fit with a Gaussian curve, and two response parameters were derived from each tuning curve. A "direction-tuning index" is defined as: $1-\left(R_{\min }-\right.$ $S) /\left(R_{\max }-S\right)$, where $R_{\max }$ and $R_{\min }$ denote the maximum and minimum values of the best-fitting Gaussian curve, and $S$ denotes the spontaneous activity level. "Preferred direction" is defined as the peak of the Gaussian curve, provided that the data exhibited significant modulation by direction of motion, as assessed by an ANOVA $(p<0.05)$.

Next, we measured a disparity-tuning curve, as illustrated in Figure $3 B$. The range of disparities tested varied from site to site according to our initial estimate of disparity tuning at the site. The data were fit with a cubic spline interpolation, and two parameters were again extracted from this curve. The "disparity-tuning index" was defined in an identical manner to the direction-tuning index described above. For recording sites exhibiting significant modulation of the response by disparity (ANOVA, $p<0.05$ ), the "preferred disparity" was taken as the disparity at which the smooth curve peaked.

From the monocular control responses (Fig. $3 B$, labeled $L, R$ ), we also computed an "ocular imbalance index" (OII) as: OII $=2 *$ abs $\left[\left(R_{R}-\right.\right.$ $S) /\left(R_{L}-S+R_{R}-S\right)-0.5$ ], where $R_{R}$ and $R_{L}$ denote the response levels obtained when random dots are presented monocularly to the right and left eyes, respectively, and $S$ denotes the spontaneous activity level. This index will vary from zero, when responses measured through the two eyes are the same, to values near unity, when the response to one eye is much stronger than the response to the other.

Lastly, we measured an area-summation curve by varying the diameter of the stimulus aperture from 0 to $30^{\circ}$ (Fig. $3 C$ ). The data were fit by one of two methods, depending on whether surround inhibition was observed (for additional details, see DeAngelis et al., 1994). We screened for surround inhibition by comparing the maximum response with the response obtained using the largest aperture size $(t$ test). If surround inhibition was indicated ( $p<0.05$ ), as in Figure $3 C$, we fit the data with the difference of two error functions (an error function is the integral of a Gaussian), one representing the excitatory response field and the other representing an inhibitory surround. From the fitted curve, we then defined two parameters; "optimal size" is the value at which the curve peaks (Fig. $3 C$ ), and "percent surround inhibition" is the amount by 
which the response is reduced at large sizes, measured as a percent of the peak response. If the initial screening indicated no surround inhibition $(t$ test, $p>0.05$ ), we fit the data with a single error function, and we defined the RF size to be the SD of the underlying Gaussian distribution.

\section{RESULTS}

MU recordings were made at 411 recording sites in area MT of two monkeys (S and P). Of these, 279 were made along 22 oblique penetrations (see Materials and Methods), 13 in monkey $\mathrm{S}$ and 9
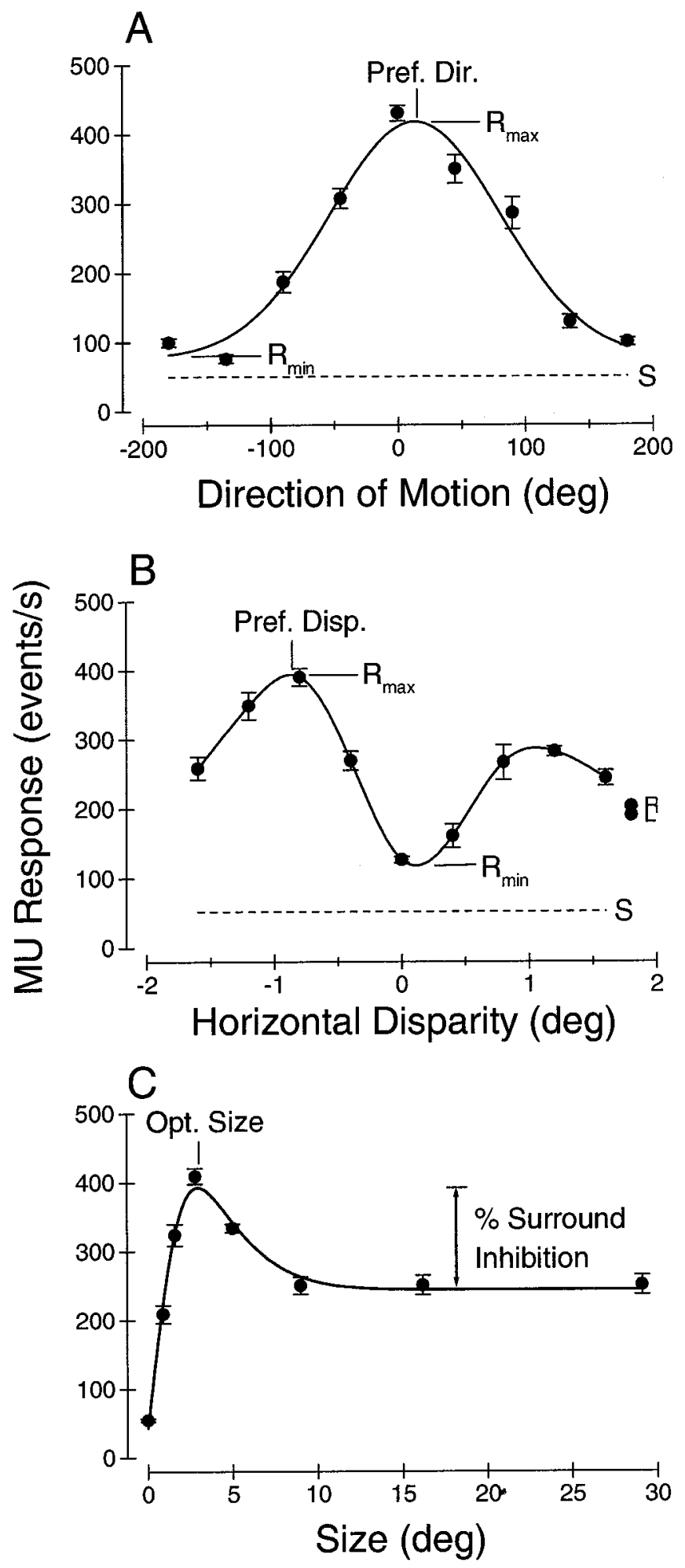

in monkey $\mathrm{P}$, and 132 recordings were made along 14 normal penetrations in monkey $P$. At each recording site, we made the measurements illustrated in Figure 3: a direction-tuning curve, a disparity-tuning curve, and an area-summation curve. Whenever possible, we also recorded the spike times of isolated SUs, in addition to the MU hash. These simultaneous MU and SU recordings allowed us to determine whether neurons in MT are clustered by disparity preference, analogous to the well known clustering of MT neurons according to direction preference (Albright et al., 1984).

\section{Clustering of disparity-selective neurons in MT}

We obtained simultaneous recordings of both MU and SU activity at 110 of 411 recording sites. Figure 4 shows disparity-tuning data from six illustrative recordings. In each case, we attempted to exclude SU spikes from the MU response (see Materials and Methods), so that the MU response reflects the combined activity of several other nearby SUs. If SU tuning generally matches MU tuning, we may conclude that nearby MT neurons have similar disparity selectivity.

Figure $4 A$ shows a case in which both MU and SU responses exhibit very strong disparity selectivity and have nearly identical tuning curves. This example is unusual in that the monocular responses of both the MU and SU activity are weak, and there is powerful binocular facilitation when the proper disparity is presented. Note that the MU response is more than an order of magnitude stronger than the SU response (ratio of response maxima $=15.6$ ). Thus, even if our window discriminator failed to exclude all SU spikes from the MU activity, the contribution of this SU would account for very little of the aggregate MU response. Across our sample of $110 \mathrm{MU}$ and SU recordings, the ratio of peak MU response to peak SU response averaged 13.5 (range, 2.9-74.5).

The examples in Figure 4, $B$ and $C$, are typical of most of our simultaneous MU and SU recordings. In both cases, the MU response is not as strongly modulated by disparity as the SU response. In Figure $4 B$, for example, the disparity-tuning indices (see Materials and Methods) for MU and SU activity are 0.53 and 0.80 , respectively. Although MU tuning is weaker, both tuning curves have similar shapes and nearly identical preferred disparities. The examples of Figure $4, B$ and $C$, are also typical in that the tuning curves have a well defined peak that is centered to the right $(B)$ or the left $(C)$ of zero disparity. Thus, these tuning curves are similar to the "tuned-near" and "tuned-far" types described by Poggio et al. (1988). In contrast, the tuning curves

\footnotetext{
$\leftarrow$

Figure 3. An example data set from one recording site. $A$, Directiontuning curve. Filled circles show the mean multiunit $(M U)$ response $( \pm 1$ $\mathrm{SE}$ ) to eight different directions of motion, $45^{\circ}$ apart. Each direction was presented four times, and trials were block randomized. The solid curve is the best-fitting Gaussian. $R_{\max }$ and $R_{\min }$ denote the maximum and minimum values of the Gaussian curve. The dashed line represents the spontaneous activity level $(S)$. The preferred direction (Pref. Dir.) is defined as that at which the Gaussian has its peak. $B$, Disparity-tuning curve. Conventions are as described in $A$, except that the solid curve is a cubic spline interpolation. Filled symbols labeled $L$ and $R$ (right) indicate the responses obtained during monocular controls when dots were presented to only the left or right eye, respectively. $C$, Area-summation curve. The solid curve here is the best fit of a difference of two error functions (see text for details). Optimal size (Opt. Size) is that at which the smooth curve has its peak, and percent surround inhibition measures the attenuation of the response at large sizes relative to the peak response. The response at size zero is equivalent to spontaneous activity.
} 
Figure 4. Comparison of disparity-tuning curves recorded simultaneously from single-unit spikes (open circles; right $y$-axis) and multiunit activity (filled circles; left $y$-axis) at six representative sites in MT. The smooth curves in each panel were generated by cubic spline interpolation. Each datum is the mean of four or five stimulus repetitions, and error bars indicate SE. The solid and dashed horizontal lines denote the spontaneous activity levels for multiunit $(M U)$ and single-unit $(S U)$ responses, respectively. Symbols $L$ and $R$ denote responses to the same moving random-dot pattern when presented monocularly to either the left or right eye, respectively. $A$, Both $M U$ and $S U$ responses exhibiting sharp disparity tuning to a narrow range of near (i.e., negative) disparities. Eccentricity (Ecc.) = 7.4 $4^{\circ}$ RF diameter (RF diam.) $=9^{\circ}$; preferred disparity $(\mathrm{PD})=-0.36^{\circ}(M U)$ or $-0.37^{\circ}(S U)$; and disparity-tuning index $(\mathrm{DTI})=1.1(M U)$ or $1.25(S U) . B$, Responses at a typical tuned-far site. Ecc. $=6.8^{\circ} ; \mathrm{RF}$ diam. $=7.5^{\circ} ; \mathrm{PD}=0.58^{\circ}$ $(M U)$ or $0.50^{\circ}(S U)$; and DTI $=0.53(M U)$ or $0.80(S U) . C$, Responses at a typical tuned-near site. Ecc. $=5.6^{\circ} ; \mathrm{RF}$ diam. $=7^{\circ} ; \mathrm{PD}=-0.07^{\circ}$ $(M U)$ or $-0.08^{\circ}(S U)$; and DTI $=0.62(M U)$ or $1.17(S U) . D$, Responses at a site with broad selectivity for far disparities. Ecc. $=8.6^{\circ}$; RF diam. $=7.5^{\circ} ; \mathrm{PD}=0.52^{\circ}(M U)$ or $0.49^{\circ}(S U)$; and DTI $=0.89(M U)$ or $1.03(S U) . E$, Both $M U$ and $S U$ at this site responsive over a broad range of near disparities. Ecc. $=10.2^{\circ}$; RF diam. $=11^{\circ}$; $\mathrm{PD}=-0.73^{\circ}(M U)$ or $-1.6^{\circ}(S U)$; and DTI $=$ $0.56(M U)$ or $0.96(S U) . F$, At this site, both $M U$ and $S U$ responses exhibiting very weak disparity tuning. Ecc. $=8.4^{\circ} ; \mathrm{RF}$ diam. $=8^{\circ}$; and DTI $=$ $0.14(M U)$ or $0.22(S U)$.
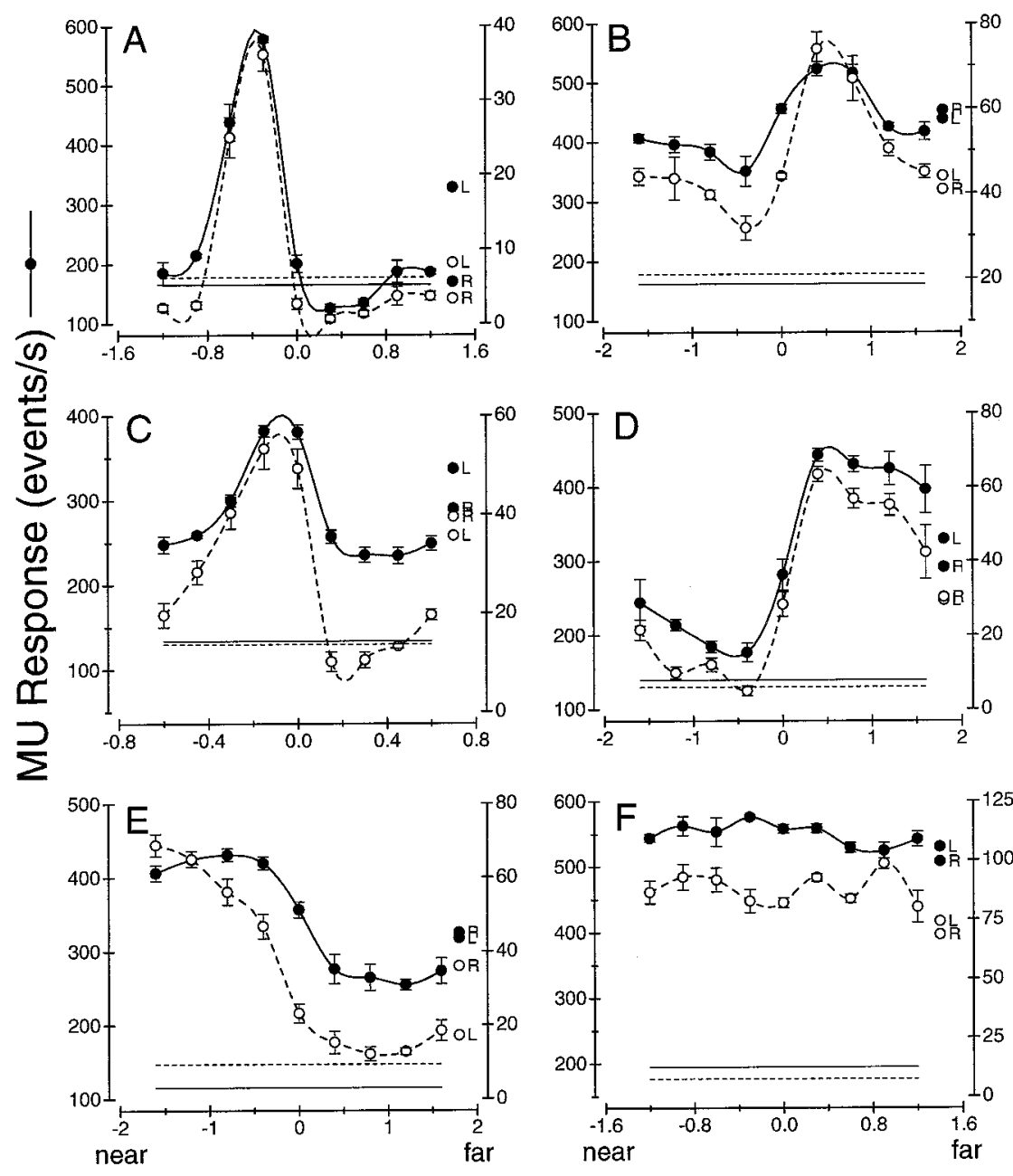

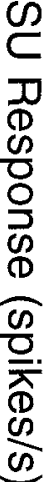

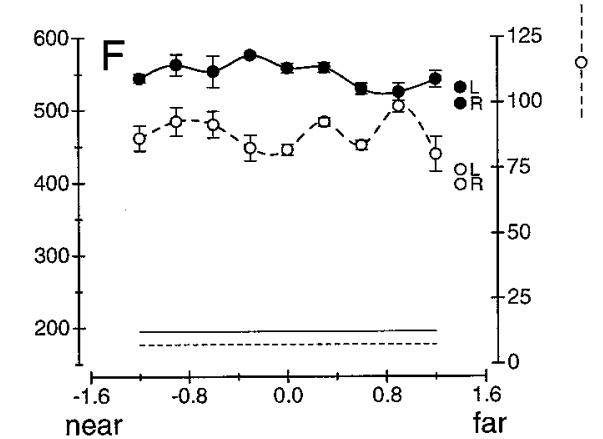

Horizontal Disparity (deg) shown in Figure 4, $D$ and $E$, have a sharp transition near zero disparity but lack well defined peaks and troughs. These examples are more similar to the "near" and "far" types of Poggio et al. (1988). For all types, however, SU and MU tunings are quite similar.

Finally, Figure $4 F$ depicts a case in which the MU disparity tuning is essentially flat (ANOVA, $p>0.05$; see Materials and Methods). Flat MU tuning could arise either because the constituent SUs also have flat tuning or because nearby SUs are tuned but to widely different disparities so that the MU tuning curve becomes flat [analogous to the situation at orientation pinwheels in cat V1 (Maldonado et al., 1997)]. The data of Figure $4 F$ support the former possibility because the SU response is also nonselective for disparity (ANOVA, $p>0.05$ ).

Figure 5 summarizes results from 110 simultaneous MU and SU recordings. Figure $5 A$ shows the relationship between disparity-tuning indices for SU and MU activity. Most data points lie above the diagonal, indicating that $\mathrm{SU}$ responses are generally more strongly tuned for disparity than are MU responses, as illustrated in Figure 4, B, $C$, and $E$. A small portion of this difference can be attributed to methodology; because SUs were excluded from the MU response, there is a series of dead times in the MU record when no MU activity can be recorded (see Materials and Methods). This tends to reduce slightly the disparity-tuning index for MU activity. More importantly, how- ever, MU and SU tuning indices are well correlated (linear regression, $r=0.67 ; p<0.0001$; slope $=0.79)$, indicating that SUs tend to exhibit weaker tuning for disparity at sites where the MU response is poorly tuned. These data argue strongly against the possibility that flat MU tuning results from a combination of SUs that are individually well tuned, but to different disparities.

Figure $5 B$ compares the preferred disparities extracted from MU and SU tuning curves. Data are plotted for the subset of recordings (77 of 110 sites) for which MU and SU tuning curves both exhibited significant selectivity (ANOVA, $p<0.05$ ). MU and SU preferred disparities agree remarkably well $(r=0.91 ; p<$ 0.0001 ; slope $=1.05)$, indicating that the MU response is an accurate predictor of the disparity preference of nearby SUs. Thus, we conclude that MT neurons are clustered according to disparity preference.

For comparison, Figure 5, $C$ and $D$, shows analogous directiontuning data from $93 \mathrm{MU}$ and SU recordings. As was the case for disparity, MU and SU direction-tuning indices are significantly correlated (linear regression, $r=0.48$; $p<0.001$; slope $=0.43$ ). Note, however, that the best linear fit (Fig. $5 A, C$, dashed lines) has a much shallower slope for the direction indices $(C)$ than for the disparity indices $(A)$. This is mainly because fewer data points fall in the lower left corner of the plot in Figure $5 C$. Among 33 sites where the MU direction-tuning index was $<0.5$, only 4 of 33 SUs $(12 \%)$ have a direction-tuning index $<0.5$. In contrast, 

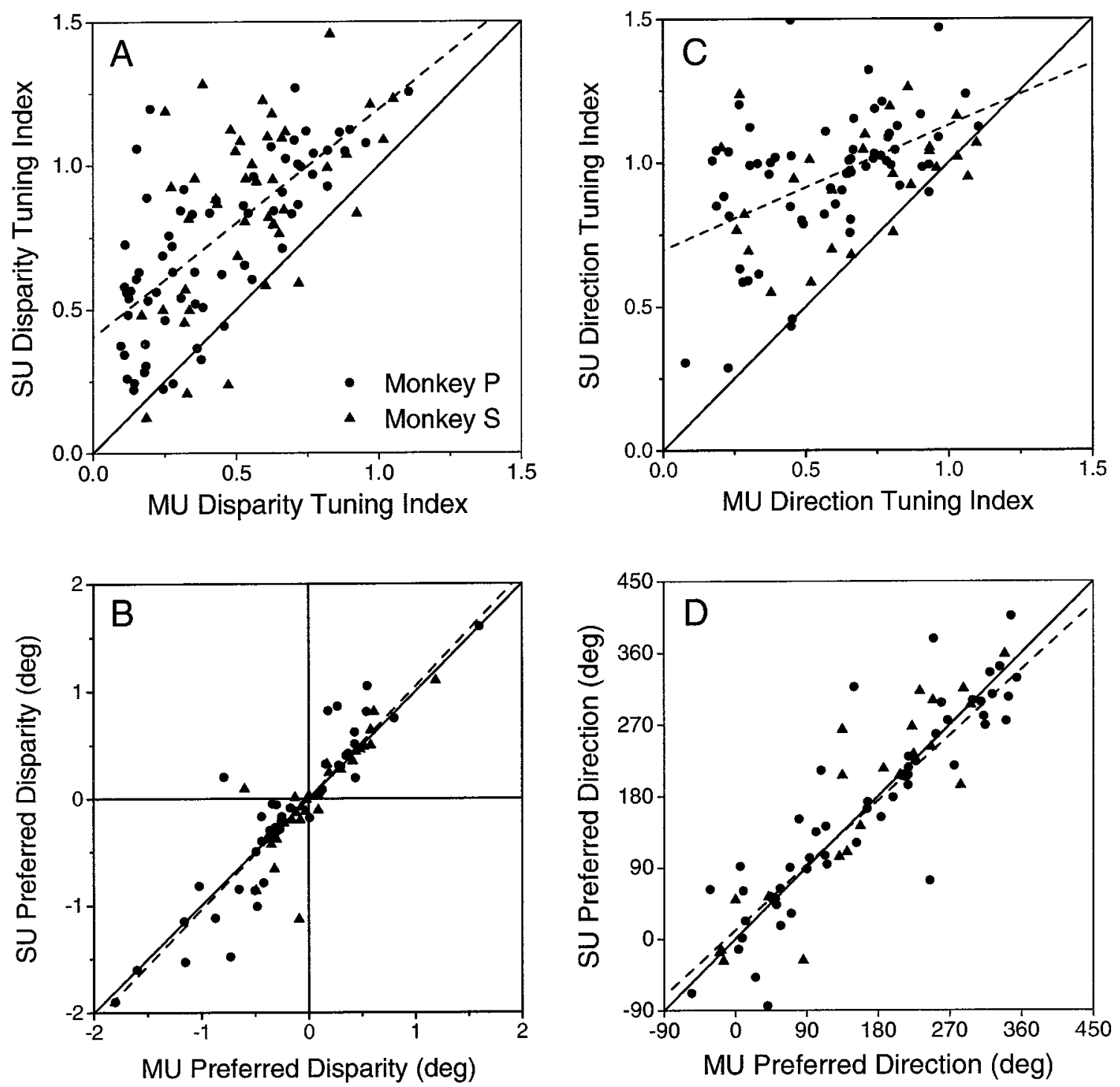

Figure 5. Quantitative comparison of disparity- and direction-tuning parameters derived from multiunit $(M U)$ and single-unit ( $S U)$ responses. In each panel, data from $S U s$ are plotted on the $y$-axis, and data from $M U$ activity are plotted on the $x$-axis. Circles and triangles represent data from monkeys $\mathrm{P}$ and $\mathrm{S}$, respectively. The solid line is the identity line, and the dashed line is the best linear fit to the data (linear regression). $A$, Comparison of disparity-tuning indices. $B$, Comparison of preferred disparities. $C$, Comparison of direction-tuning indices. $D$, Comparison of preferred directions.

among 58 sites with MU disparity-tuning indices below 0.5, 24 of 58 SUs $(41 \%)$ have indices $<0.5$ (Fig. $5 A$ ). Thus, the pattern of results for direction tuning is somewhat similar to that observed near orientation pinwheels in V1 (Maldonado et al., 1997); the aggregate (MU) response is poorly tuned, but SUs are generally well tuned to a variety of different directions. For disparity tuning, on the other hand, SUs are much more likely to exhibit poor selectivity at sites where MU tuning is poor.

Figure $5 D$ compares the preferred directions of SU and MU responses for 79 of 93 sites where both responses exhibited significant tuning (ANOVA, $p<0.05$ ). SU and MU preferences are strongly correlated $(r=0.88 ; p<0.0001$; slope $=0.92)$, as expected from the known columnar organization for direction in MT (Albright et al., 1984). Thus, Figure 5, $B$ and $D$, shows that MU responses are an excellent predictor of SU preferences, both for direction and disparity tuning.

The data presented thus far show that neurons in MT are clustered according to their disparity-tuning properties as well as their direction-tuning properties. In the next section we explore the functional organization of disparity information by measuring MU responses at regular intervals along electrode penetrations through MT.

\section{Functional architecture for binocular disparity}

MU responses were collected at 100 or $150 \mu \mathrm{m}$ intervals along electrode penetrations that were initiated from two different angles. In oblique penetrations (see Fig. $2 A$ ), the electrode traveled through MT at a shallow angle relative to the cortical surface. Because MT is organized retinotopically (Albright and Desimone, 1987; Maunsell and Van Essen, 1987), we expect to observe a progressive shift of RF position across the visual field as we move the electrode along an oblique penetration. Similarly, because MT contains a columnar architecture for direction of motion (Albright et al., 1984), we expect preferred directions to vary systematically along these penetrations [except at singularity points in the direction map (see Albright et al., 1984; Malonek et 


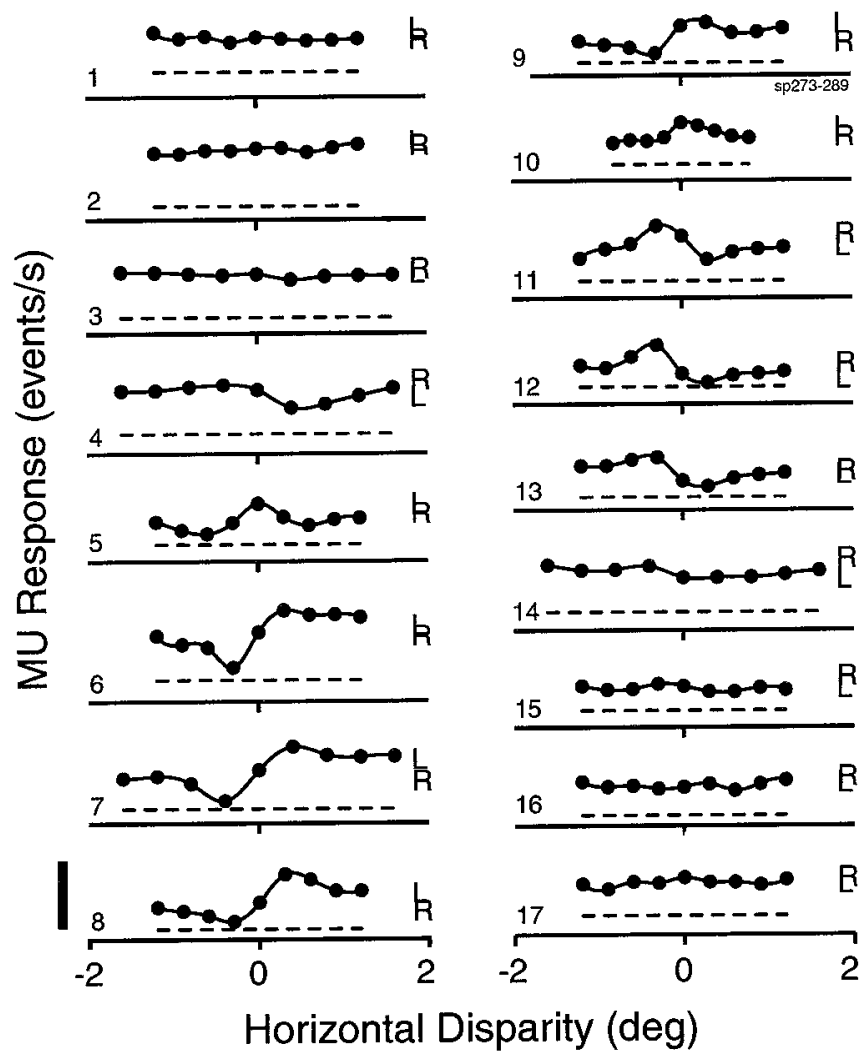

Figure 6. Sequence of disparity-tuning curves recorded along an oblique penetration through MT in monkey S. Curves are numbered in the order in which they were obtained. Each graph shows multiunit $(M U)$ response plotted against horizontal disparity. Dashed horizontal lines represent the spontaneous activity level at each site, and letters $L$ and $R$ are plotted at the response levels measured in monocular controls. Error bars indicate SE and are plotted around each mean but are generally smaller than the data points themselves. Scale bar, 400 events/sec.

al., 1994)]. These known variations in RF position and direction across the surface of MT may then be used as a reference to evaluate changes in disparity selectivity. In normal penetrations (Fig. 2), our electrode passed through MT approximately orthogonal to the cortical surface. Thus, we expect to see both RF positions and preferred directions of motion remain much more constant along these penetrations, again providing a useful reference to evaluate changes in disparity tuning.

\section{Oblique penetrations}

Figure 6 shows a sequence of disparity-tuning curves measured at $100 \mu \mathrm{m}$ intervals along an oblique penetration in monkey $\mathrm{S}$. Disparity selectivity is essentially absent at the beginning of the penetration (Fig. 6, sites 1-3) and again at the end (sites 14-17). Note, however, that all of these MU responses are well above the spontaneous activity level (Fig. 6, dashed horizontal lines). In the middle of the penetration (Fig. 6, sites 4-13), a region of strong disparity selectivity spanned $\sim 1 \mathrm{~mm}$. Within this region, the preferred disparity varied systematically with distance along the penetration. The MU activity at site 5 (Fig. 6) responded optimally to zero disparity, whereas sites 6-9 clearly preferred far disparities. A modest preference for zero disparity again appeared at site 10 (Fig. 6), but subsequent sites (11-13) responded best to near disparities.

Figure 7 summarizes quantitative data from the same penetra-
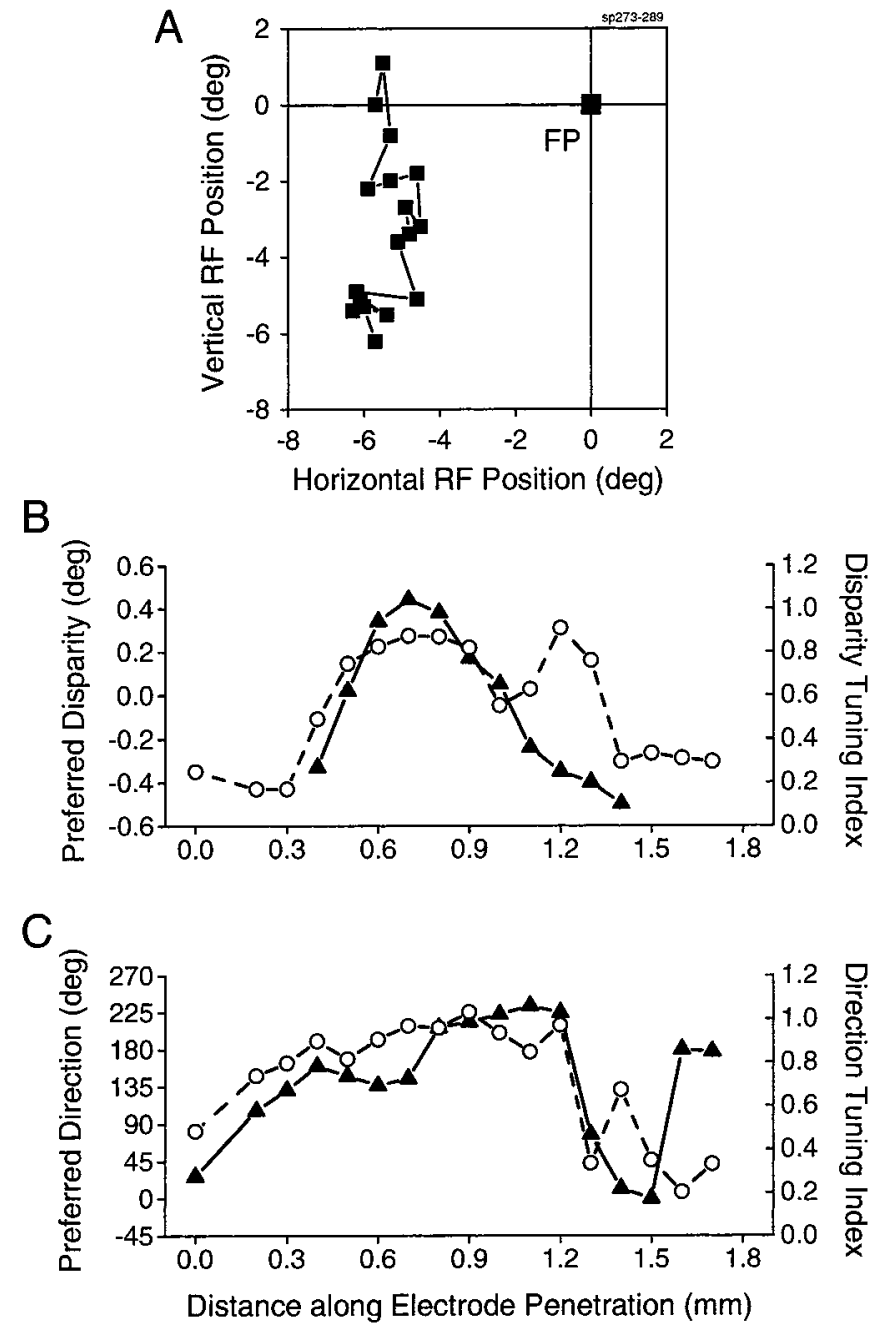

Figure 7. Quantitative summary of data recorded along the same oblique penetration illustrated in Figure 6. $A$, Location of the center of the MU receptive field $(R F)$ at each recording site. $R F$ location was estimated using a computerized search program (see Materials and Methods) and is plotted relative to the fixation point $(F P)$ that has coordinates $(0,0)$. The sequence of $R F$ locations begins in the lower left quadrant and moves upward toward the horizontal meridian. $B$, Changes in disparity tuning quantified by plotting the disparity-tuning index (open circles; right $y$-axis) and the preferred disparity (filled triangles; left $y$-axis) as a function of distance along the electrode penetration. Note that preferred disparities are not plotted for sites where disparity tuning was not statistically significant (ANOVA, $p>0.05$ ). $C$, Quantitative summary of changes in direction tuning. Direction-tuning index (open circles; right $y$-axis) and preferred direction of motion ( filled triangles; left $y$-axis) are plotted in the same format used in $B$.

tion. Figure $7 A$ shows that the spatial location of the MU RF moved gradually from the lower left quadrant of the visual field up to the horizontal meridian during the penetration, a typical progression for one of our oblique penetrations through MT. Figure $7 B$ plots the disparity-tuning index (open circles; right $y$-axis) and the preferred disparity (filled triangles; left $y$-axis) as a function of distance along the penetration. These disparity parameters were extracted from the data of Figure 6, as described in Materials and Methods. There are two salient features in these data. First, the disparity-tuning index is small at the beginning and end of the penetration but is large over a $1 \mathrm{~mm}$ span in the middle. Thus, disparity-selective neurons appear to have a patchy 


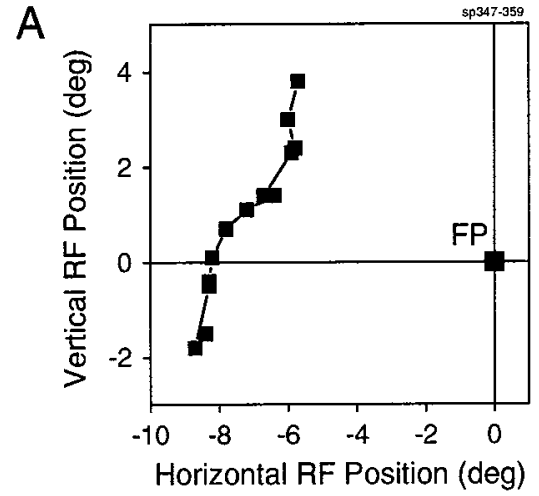

$\mathrm{B}$

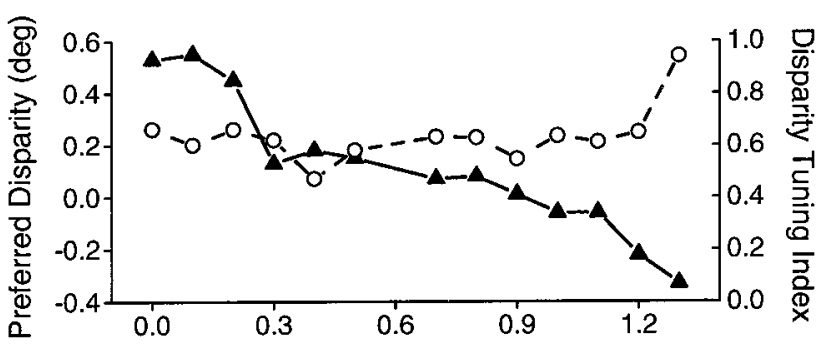

$\mathrm{C}$

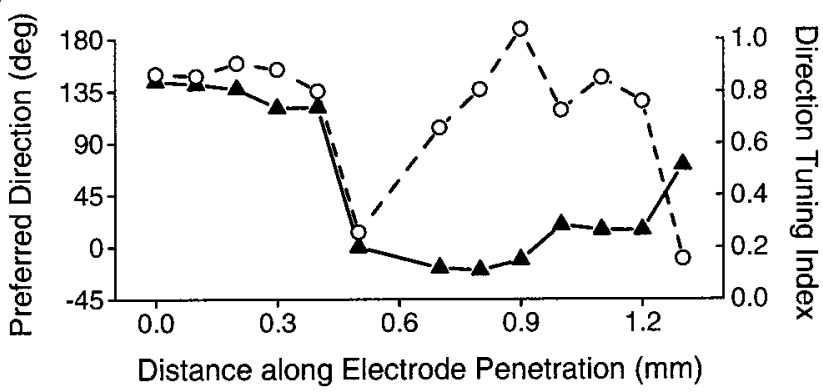

Figure 8. Quantitative data summary for another oblique penetration from monkey S. The format of this figure is identical to that of Figure 7.

distribution within MT. Second, within the central region of strong tuning, the preferred disparity changes smoothly from near to far and back to near. This observation suggests that there is a systematic map of binocular disparity within this region of MT.

For comparison, Figure $7 C$ shows direction-tuning data, plotted in the same manner, for this penetration. Over the first 1.2 $\mathrm{mm}$ of the penetration, the direction tuning is consistently strong, and the preferred direction changes gradually from $\sim 45$ to $225^{\circ}$. Near the end of the penetration, the direction-tuning index drops precipitously, and the preferred direction jumps by $\sim 180^{\circ}$ from the 1.2 to the $1.3 \mathrm{~mm}$ mark in the penetration. This behavior near the end of the penetration is consistent with our electrode encountering a discontinuity point in the direction map (see Albright et al., 1984; Malonek et al., 1994). In this penetration, the direction discontinuity approximately coincides with the transition from strong to weak disparity selectivity (Fig. $7 B$ ), but this was not generally the case, as illustrated in the next example.

Figure 8 illustrates data from another oblique penetration through MT. Figure $8 A$ shows that RF locations again move progressively from the lower hemifield to the upper hemifield. Figure $8 B$ shows that the disparity-tuning index (open circles) remains constant at $\sim 0.6$ throughout the penetration, indicating that the electrode encountered a patch of MT with moderately

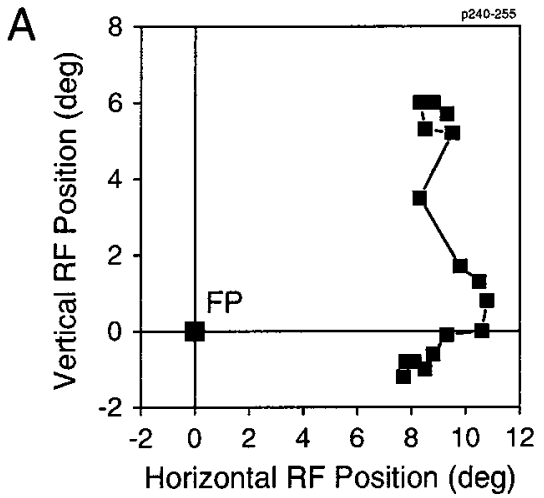

B
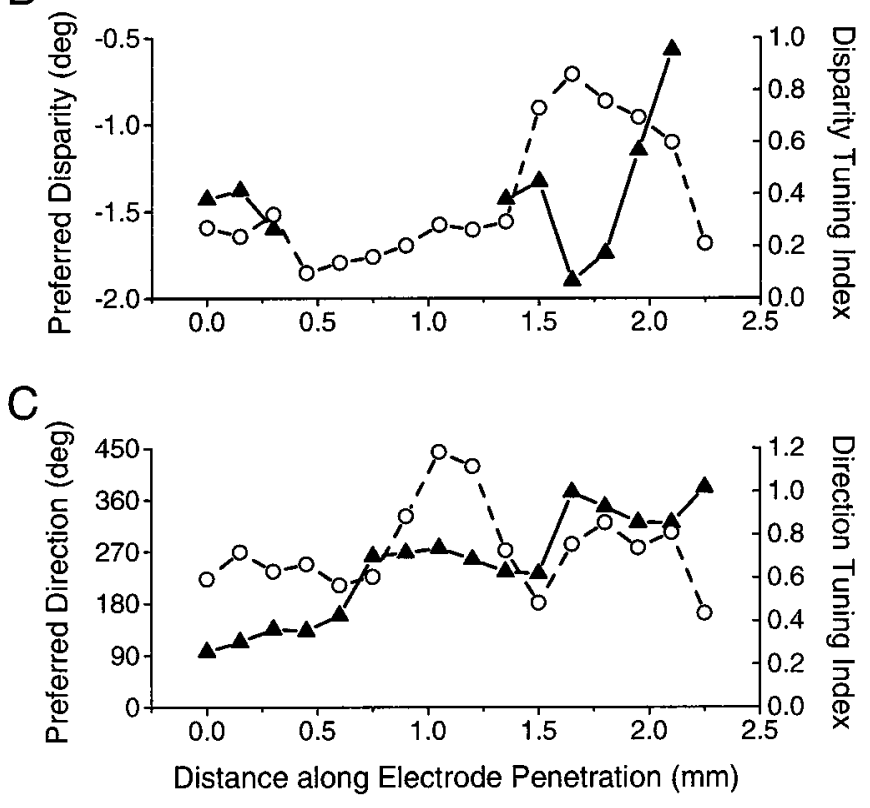

Figure 9. Quantitative data summary for an oblique penetration from monkey P. Note that the disparity tuning is very weak for $>1 \mathrm{~mm}$ at the beginning of the penetration, after which there is a region of strong disparity selectivity. The format of this figure is identical to that of Figure 7.

strong disparity selectivity. Within this penetration, the preferred disparity (Fig. $8 B$, filled triangles) changes gradually from far to near, again suggesting a systematic map of disparity. Figure $8 C$ shows that direction tuning can exhibit a discontinuity with no corresponding discontinuity in disparity selectivity. At $0.5 \mathrm{~mm}$ from the start of the penetration, the direction-tuning index fell abruptly, and the preferred direction jumped by $\sim 135^{\circ}$. Note, however, that there is no corresponding discontinuity at this location in either the disparity-tuning index or the preferred disparity (Fig. 8B). The direction-tuning index dipped sharply for a second time at the last recording site along this penetration, but we were unable to continue the penetration beyond this point because the monkey ceased to perform fixation trials. As this penetration suggests, we generally did not observe any correlation between the occurrence of discontinuities in direction and disparity selectivity.

Figure 9 shows data from a third oblique penetration. This penetration illustrates a feature that we observed in several experiments; regions of poor disparity selectivity could last up to 1 mm or more. Figure $9 B$ shows that the disparity-tuning index remains below 0.3 over the first $1.35 \mathrm{~mm}$ of the penetration. This 


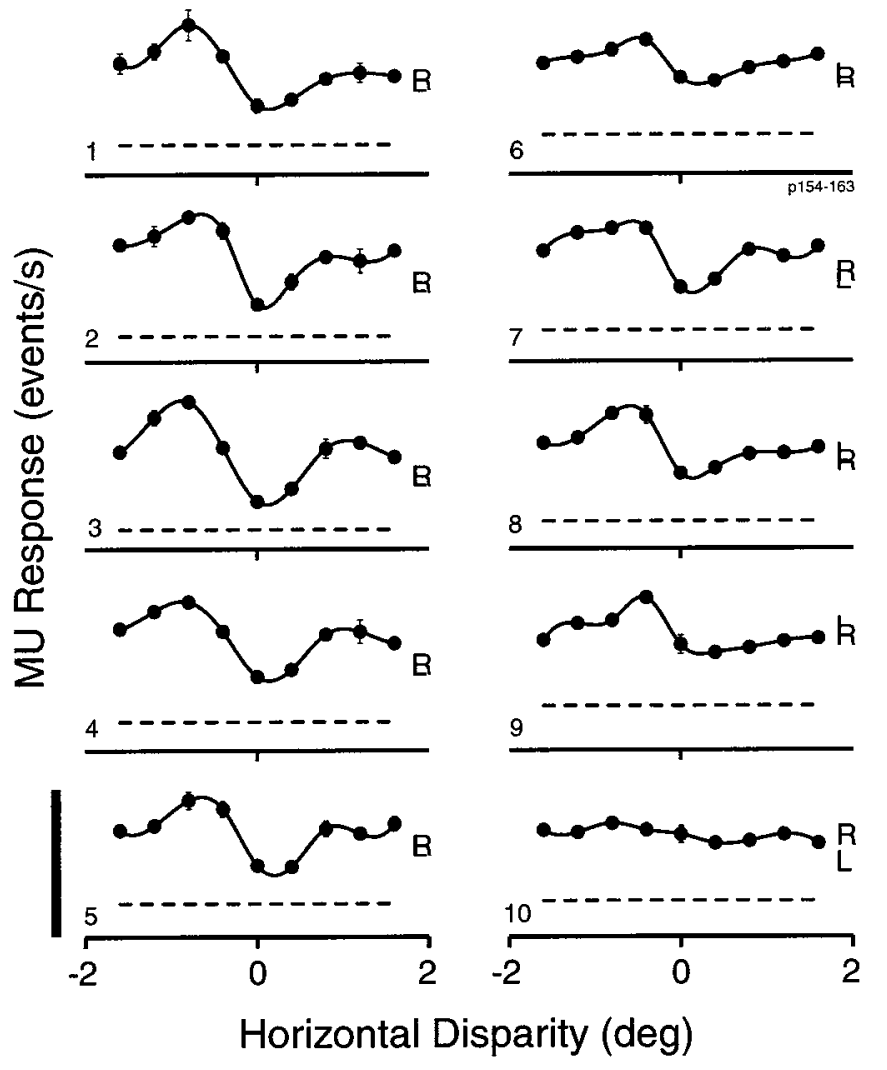

Figure 10. Sequence of disparity-tuning curves recorded at $150 \mu \mathrm{m}$ intervals along a normal penetration from monkey P. Conventions are described in Figure 6. Note that almost all disparity-tuning curves have a very similar shape, with a preference for near disparities. Scale bar, 400 events/sec.

extended region of poor disparity tuning is then followed by a stretch of strong selectivity that lasts for $0.6 \mathrm{~mm}$, before the disparity tuning again becomes poor at the end of the penetration. Within the region of strong tuning, the preferred disparity changes from large near to small near. Figure $9 C$ shows that direction tuning is moderate to strong throughout this penetration and that the preferred direction of motion increases from $\sim 90$ to $360^{\circ}$ in an uncommon step-like manner. This penetration is representative in that we often encountered long stretches of poor disparity selectivity in oblique penetrations, but we seldom observed regions of poor direction tuning that lasted more than a few hundred micrometers. We shall return to this point below.

It is clear from Figures 7-9 that disparity tuning changes in a systematic manner along oblique penetrations through MT, although the pattern of variation differs substantially from one penetration to the next. Given that our electrode did not travel strictly tangential to the cortical surface in these oblique penetrations (see Materials and Methods; Fig. 2A), however, some of this variation could be laminar in origin. To address this issue, we examined penetrations that entered MT approximately normal to the cortical surface.

\section{Normal penetrations}

Figure 10 shows a sequence of disparity-tuning curves recorded at $150 \mu \mathrm{m}$ intervals along a normal penetration through MT. The tuning curves at most sites have a shape that is intermediate between the "tuned-near" and "tuned-inhibitory" types of Poggio et al. (1988). The peak at near disparities is clearly larger than the

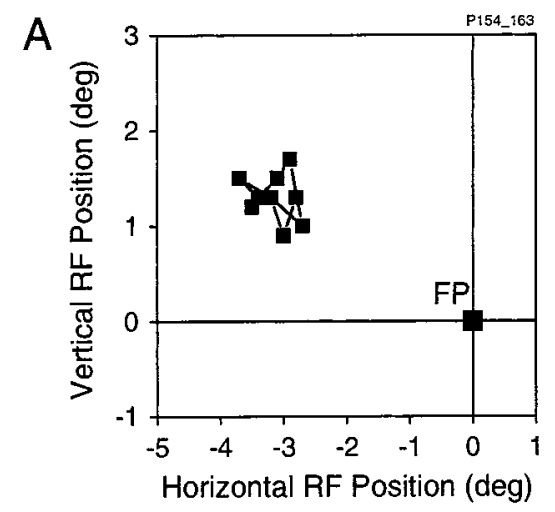

B
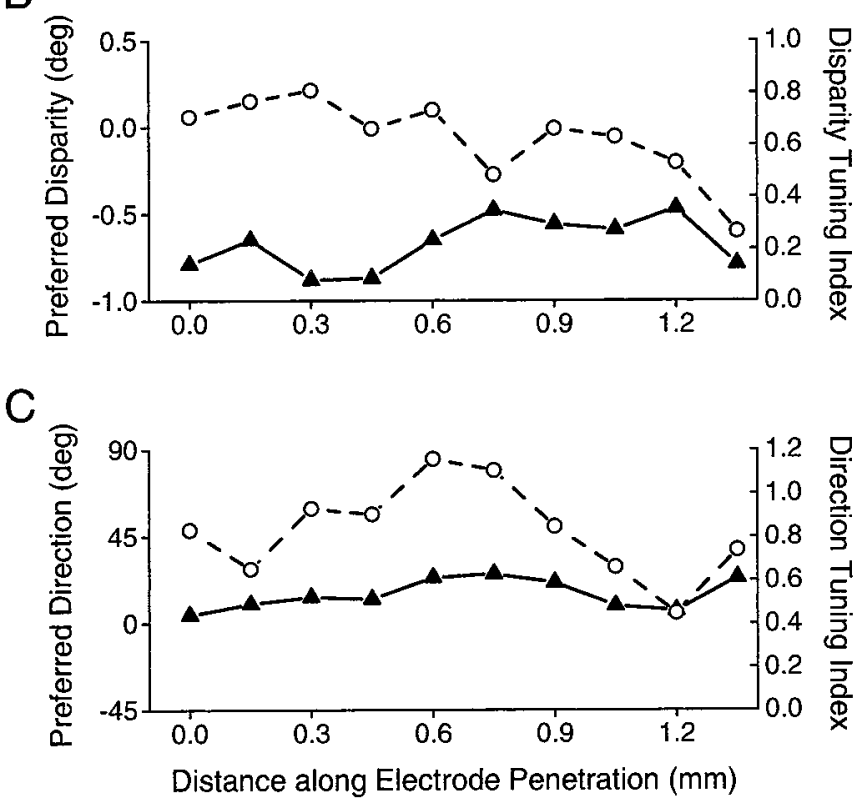

Figure 11. Quantitative data summary for the normal penetration of Figure 10. The format of this figure is identical to that of Figure 7.

peak at far disparities, but there is also a clear trough that is centered at zero disparity in most cases. Thus, these disparitytuning curves, like many of those that we have recorded, do not fall cleanly into one of the six classes defined by Poggio et al. (1988) (see Discussion for more on this point). More importantly, Figure 10 shows that disparity tuning remains approximately constant along this normal penetration, consistent with the possibility that disparity is organized in vertical columns.

Figure 11 summarizes the data from this normal penetration. Figure $11 A$ shows that the location of the MU RF is quite consistent across sites in this penetration. This constancy of RF location supports the histological evidence that penetrations made through the frontally located cylinder penetrated MT approximately normal to the cortical surface (Fig. 2B). Also in agreement with a normal approach, Figure $11 C$ shows that the preferred direction of motion remains quite constant throughout the penetration, although the direction-tuning index varies somewhat. Similarly, Figure $11 B$ shows that the preferred disparity stays relatively constant between -0.5 and $-0.9^{\circ}$ of disparity. The disparity-tuning index also remains fairly constant, except at the last recording site that is weakly tuned. This last site occurred at the boundary between gray and white matter, perhaps accounting for the reduced selectivity. 


\section{Quantitative analyses}

To quantify the variation of tuning parameters along oblique penetrations and to assess the differences between oblique and normal penetrations, we adapted an analysis used previously by LeVay and Voigt (1988) to study the organization of disparity selectivity in cat striate cortex. In this analysis, we examine how the magnitude of the change in a particular parameter (e.g., preferred disparity) varies with the distance between a pair of recording sites. If a parameter such as preferred direction or preferred disparity is mapped systematically across the surface of MT, the values of the parameter should be more similar for nearby recording sites than for distant recording sites (in oblique or tangential penetrations).

Filled circles in Figure $12 \mathrm{~A}$ show this relationship for measurements of preferred disparity obtained along oblique penetrations through MT. Each datum gives the mean absolute difference in preferred disparity $\mid \Delta$ preferred disparity $\mid$ for all recording sites that were separated by a given distance. We computed $\mid \Delta$ preferred disparity| for each unique pair of recording sites in each penetration, and the data were then pooled across all 22 oblique penetrations from the two monkeys. Two features of the data in Figure $12 A$ should be noted. First, | $\Delta$ preferred disparity| increases smoothly with the distance between recording sites, indicating that preferred disparities are indeed more similar for nearby sites than for more distant sites. Second, as the distance between recording sites increases, the curve (Fig. 12A, filled circles) asymptotes near the solid horizontal line, which represents the average value of $\mid \Delta$ preferred disparity $\mid$ that one would obtain by randomly drawing pairs of recording sites from the entire population (we computed this value by performing exactly this procedure; random pairings were drawn several thousand times by computer from the overall distribution of recording sites). For recording sites separated by more than $\sim 0.5 \mathrm{~mm}$, therefore, the difference in preferred disparity approaches the value that would be expected if the organization of disparity-selective neurons in MT were random. Thus the map of disparity in MT is orderly within local neighborhoods, perhaps $1.0 \mathrm{~mm}$ in diameter. The disparity map has no detectable regularity on a more global scale, as might be expected of a periodically repeating map.

Filled triangles in Figure $12 \mathrm{~A}$ show the change in $\mid \Delta$ preferred disparity| with distance for normal penetrations. Again, the average value of $\mid \Delta$ preferred disparity $\mid$ increases with distance, as expected because most of our penetrations were not precisely normal to the cortical surface (see Materials and Methods). The observed values change less rapidly than do those from oblique penetrations, however, and do not approach the solid horizontal line (Fig. 12A), even for separations as large as $1 \mathrm{~mm}$. Thus preferred disparity varies substantially less in normal than in oblique penetrations, and the average $\mid \Delta$ preferred disparity $\mid$ is always well below the value expected by chance. We were initially surprised that this curve showed any tendency at all to rise and saturate for larger separations. Re-examination of the normal penetrations, however, revealed a nontrivial amount of heterogeneity among individual penetrations. For a few penetrations, $\mid \Delta$ preferred disparity| increased rapidly with distance and saturated near the solid horizontal line (Fig. 12A) (similar to an oblique penetration). Presumably, our electrodes did not travel normal to the surface of MT in these cases, because RF position and preferred direction also changed systematically. This is not surprising because MT is somewhat folded within the superior temporal sulcus. In several other penetrations, the curve plotting
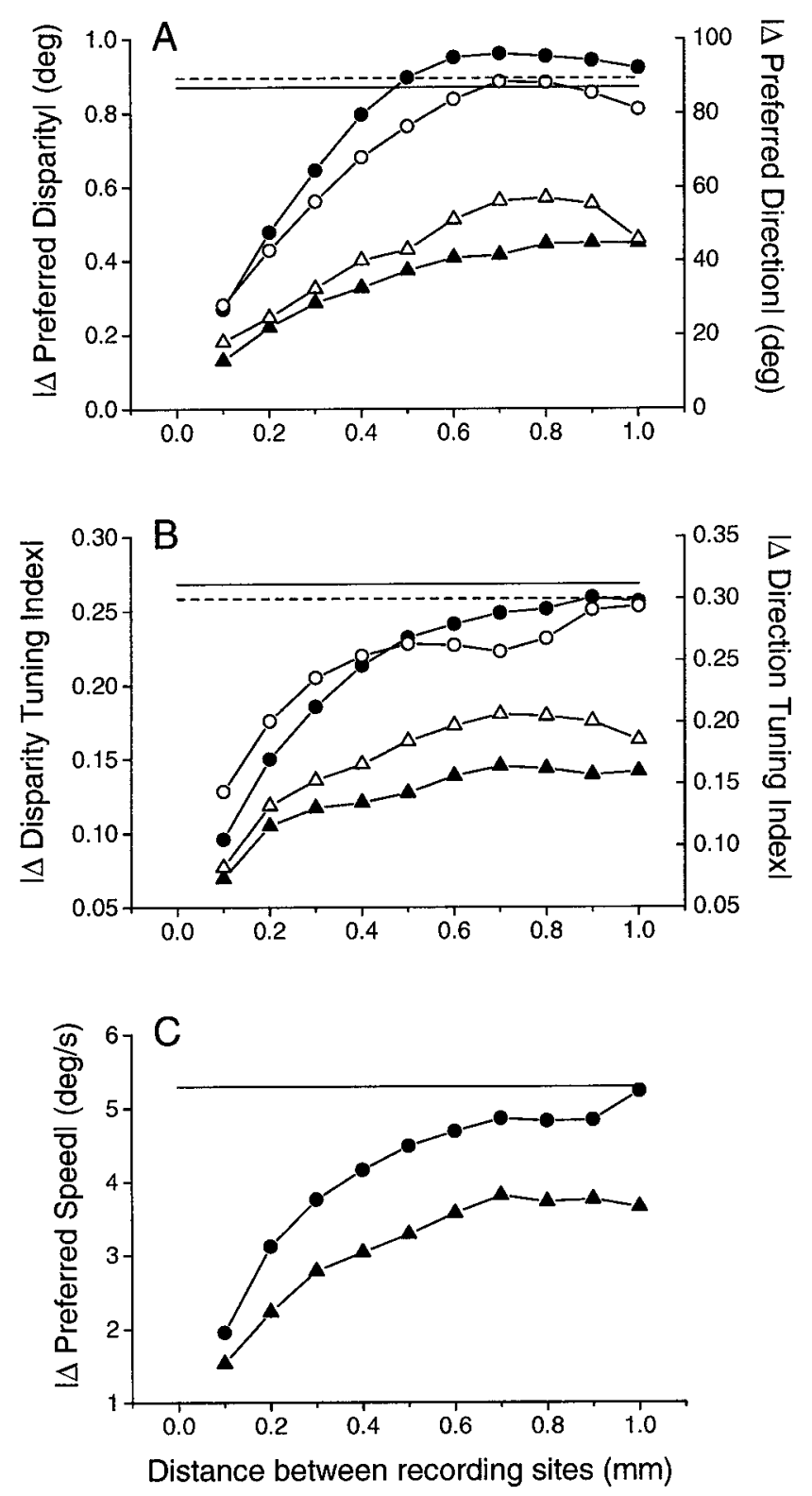

Figure 12. Quantitative summary of the functional organization of disparity, direction, and speed tuning in MT. In each panel, circles are data derived from oblique penetrations, and triangles are data from normal penetrations. Filled symbols correspond to the parameter plotted on the left $y$-axis; open symbols correspond to the parameter plotted on the right $y$-axis. A, Filled circles and triangles show the average absolute difference in preferred disparity $\mid \Delta$ Preferred Disparity $\mid$ between pairs of recording sites as a function of distance between the sites. The solid horizontal line gives the value of $\mid \Delta$ Preferred Disparity $\mid$ expected from drawing random pairs of sites from the entire population. Open circles and triangles show the analogous data for preferred direction of motion $\mid \Delta$ Preferred Direction|. The dashed horizontal line gives the value of $\mid \Delta$ Preferred Direction $\mid$ expected from random pairings. The left and right $y$-axes have been scaled to round numbers so that the solid and dashed horizontal lines are approximately superimposed. $B$, Data are shown in the same format for the disparity-tuning index ( filled symbols and solid horizontal line; left $y$-axis) and the direction-tuning index (open symbols and dashed horizontal line; right $y$-axis). $C$, Similar data are shown for the preferred speed of motion.

$\mid \Delta$ preferred disparity $\mid$ against distance was nearly flat. This heterogeneity among penetrations explains why the average curve (Fig. 12A, filled triangles) shows a gradual rise and some saturation. 
To quantify the trends apparent in these data, we performed a two-way ANOVA with distance between recording sites and penetration type (oblique vs normal) as the two factors. For the preferred disparity data in Figure $12 A$ (filled circles and triangles), both the main effect of distance $\left[F_{(9,2708)}=12.61 ; p \ll 0.001\right]$ and the main effect of penetration type $\left[F_{(1,2708)}=122.95 ; p \ll 0.001\right]$ are highly significant. The interaction between these two factors is also significant $\left[F_{(9,2708)}=2.12 ; p=0.025\right]$, indicating that $\mid \Delta$ preferred disparity $\mid$ increases more rapidly with distance in oblique penetrations than in normal penetrations.

Open symbols in Figure $12 \mathrm{~A}$ show analogous data for the preferred direction of motion. Note that the left and right $y$-axes in Figure $12 A$ are scaled (to the nearest round numbers) so that the values expected from random pairings (solid and dashed horizontal lines) are approximately superimposed. This allows changes in $\mid \Delta$ preferred disparity $\mid$ and $\mid \Delta$ preferred direction $\mid$ with distance to be compared directly. The pattern of results is very similar to that for preferred disparity, suggesting that these two parameters are organized similarly. The main effect of distance is highly significant for preferred direction $\left[F_{(9,2946)}=46.58 ; p \ll\right.$ $0.001]$, as is the main effect of penetration type $\left[F_{(1,2946)}=349.4\right.$; $p \ll 0.001]$. Thus, both preferred direction and disparity vary much less rapidly in normal penetrations than in oblique penetrations. The interaction between distance and penetration type is also significant for preferred direction $\left[F_{(9,2946)}=4.1 ; p<\right.$ $0.001]$. Interestingly, the rise in $\mid \Delta$ preferred direction $\mid$ with distance follows a similar trajectory to that of $\mid \Delta$ preferred disparity $\mid$ (Fig. 12A, compare filled and open circles). This similarity for oblique penetrations suggests that preferred direction and disparity vary across the surface of MT on a similar spatial scale. If, for example, direction varied much more rapidly than disparity, then the curve for $\mid \Delta$ preferred direction $\mid$ should rise much more rapidly with distance than the curve for $\mid \Delta$ preferred disparity $\mid$. Clearly this is not the case.

Figure $12 B$ shows similar data for both the disparity-tuning index (filled symbols) and the direction-tuning index (open symbols). In both cases, the main effect of distance is highly significant $\left[F_{(9,3060)}>100.0 ; p \ll 0.001\right]$, as is the main effect of penetration type $\left[F_{(1,3060)}>18.0 ; p \ll 0.001\right]$. The interaction between the two factors is significant for the disparity-tuning index $\left[F_{(9,3060)}=\right.$ $3.59 ; p<0.001]$ but not for the direction-tuning index $\left[F_{(9,3060)}=\right.$ $0.47 ; p=0.89]$. These data show that the strength of tuning of MU activity varies smoothly across the surface of MT, both for direction of motion and binocular disparity. Similar variations in tuning strength have been observed in studies using optical imaging techniques. For example, Malonek et al. (1994) have observed gradual variations in direction selectivity across the surface of MT in the owl monkey.

In most experiments, we estimated the preferred speed of MU activity at each recording site, in addition to measuring both direction and disparity tuning. Unlike the measurements of direction and disparity tuning, however, these estimates of speed preference were qualitative. At each site, we assessed speed tuning using a computerized search program, as described in Materials and Methods. Figure $12 C$ shows the analysis of these speed data. As for the direction and disparity parameters, there is a highly significant increase in $\mid \Delta$ preferred speed $\mid$ with distance between the recording sites $\left[F_{(9,3060)}=15.98 ; p \ll 0.001\right]$. This indicates that neurons are clustered in MT according to speed preference, as reported previously by Maunsell and Van Essen (1983a). In addition, our analysis shows that there is a significant main effect of penetration type $\left[F_{(1,3060)}=41.46 ; p \ll 0.001\right]$, indicating that preferred speed varies less in normal penetrations than in oblique penetrations. There was no significant interaction between distance and penetration type $\left[F_{(9,3060)}=0.39 ; p=\right.$ 0.94]. Thus, with the caveat that our estimates of speed preference are not quantitative, it seems that MT contains a columnar organization for speed as well as direction and disparity, as suggested originally by Maunsell and Van Essen (1983a).

Eccentricity could be a confounding factor in the data analysis of Figure 12. Preferred speed, for example, is known to increase with eccentricity (Maunsell and Van Essen, 1983a). If $\mid \Delta$ eccentricity| generally increases with distance between recording sites in our penetrations, then the apparent increase in $\mid \Delta$ preferred speed| with distance could be attributable to the confounding effect of eccentricity. To exclude this possibility, we incorporated $\mid \Delta$ eccentricity $\mid$ as a covariate into our two-way ANOVA. The main effects of distance and penetration type described above remained highly significant $(p \ll 0.001)$ when eccentricity was incorporated into the model; thus, these effects cannot be accounted for by eccentricity.

\section{Spatial distribution of disparity-tuned patches}

The quantitative analyses described thus far fail to capture two salient differences between the organization of direction and disparity selectivity in MT. First, direction selectivity is more prevalent, on average, than disparity selectivity. Figure $13 A$ shows distributions of the direction-tuning index, measured from MU responses, for each of the two monkeys. Mean values are 0.75 for monkey $\mathrm{S}$ and 0.59 for monkey P. Similarly, Figure $13 B$ shows the distributions of disparity-tuning indices; mean values are 0.56 for monkey $\mathrm{S}$ and 0.42 for monkey P. For each monkey, the average direction-tuning index is significantly larger than the average disparity-tuning index (Mann-Whitney $U$ test, $p<0.001$ ). This difference is consistent with single-unit studies, which show that $\sim 90 \%$ of MT neurons are direction selective (Zeki, 1978b; Maunsell and Van Essen, 1983a; Albright, 1984), whereas only two-thirds are disparity selective (Maunsell and Van Essen, 1983b).

Second, as suggested by the data from individual penetrations in Figures 7 and 9, disparity-tuned neurons are clustered into discrete patches in the middle of regions of MT that are poorly tuned for disparity. To estimate quantitatively the spatial scale of this patchy structure, we subdivided all oblique penetrations into segments that were poorly tuned for disparity (DTI $<0.5$ for each recording site) or well tuned for disparity (DTI $>0.5$ for each recording site). Figure $13, D$ and $F$, shows frequency histograms of the lengths of the poorly tuned and well tuned segments, respectively, for each of the two monkeys. For monkey P, well tuned segments were shorter, on average, than poorly tuned segments. Thus, regions of MT that are well tuned for disparity appear to form "islands," typically 300-700 $\mu \mathrm{m}$ wide, positioned within a larger matrix of poorly tuned cortex. For monkey S, the distributions of segment lengths were similar for weak and strong tuning. As in monkey $\mathrm{P}$, well tuned patches appear to be 300-700 $\mu \mathrm{m}$ wide, on average, but the regions of poorly tuned cortex were smaller in monkey $\mathrm{S}$ than in monkey $\mathrm{P}$. Figure 13, $C$ and $E$, displays analogous data for regions of MT that were poorly tuned and well tuned, respectively, for direction of motion. The directional data contrast strikingly with the disparity data: well tuned segments were substantially longer than poorly tuned segments for both monkeys. Thus the general spatial organization is reversed for directionality, with poorly tuned patches forming small islands within a general matrix that is highly directional. It is 
DIRECTION
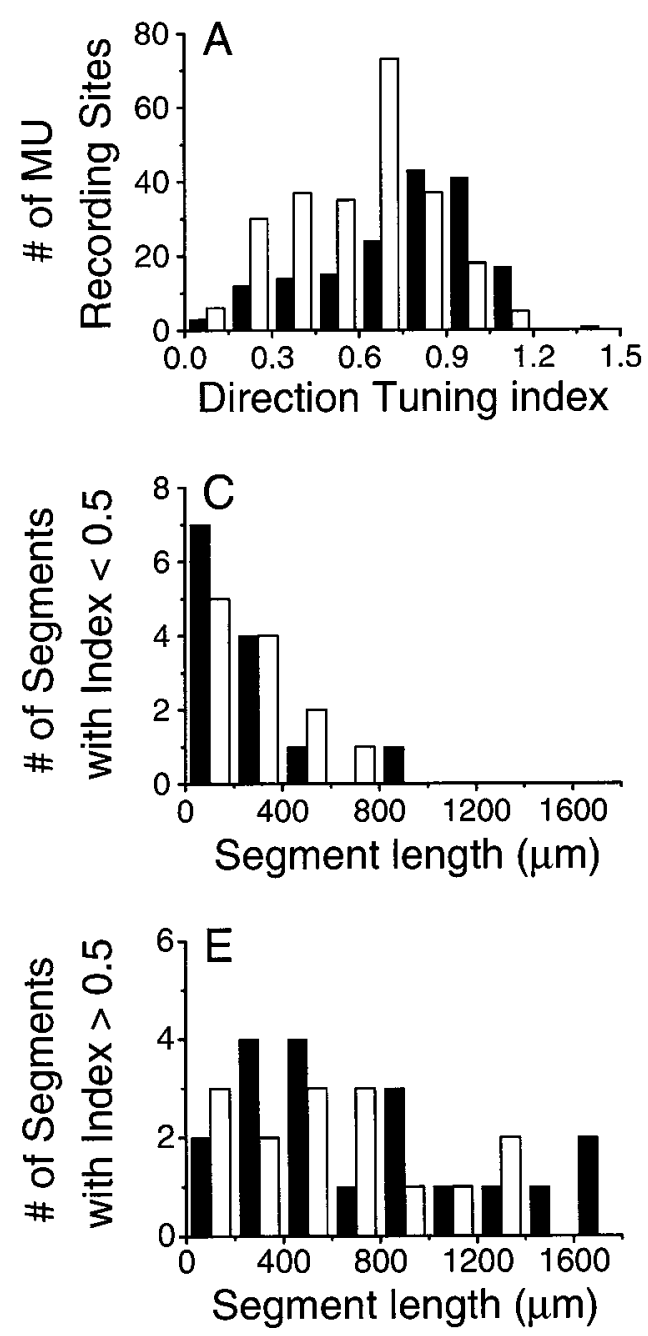

\section{DISPARITY}
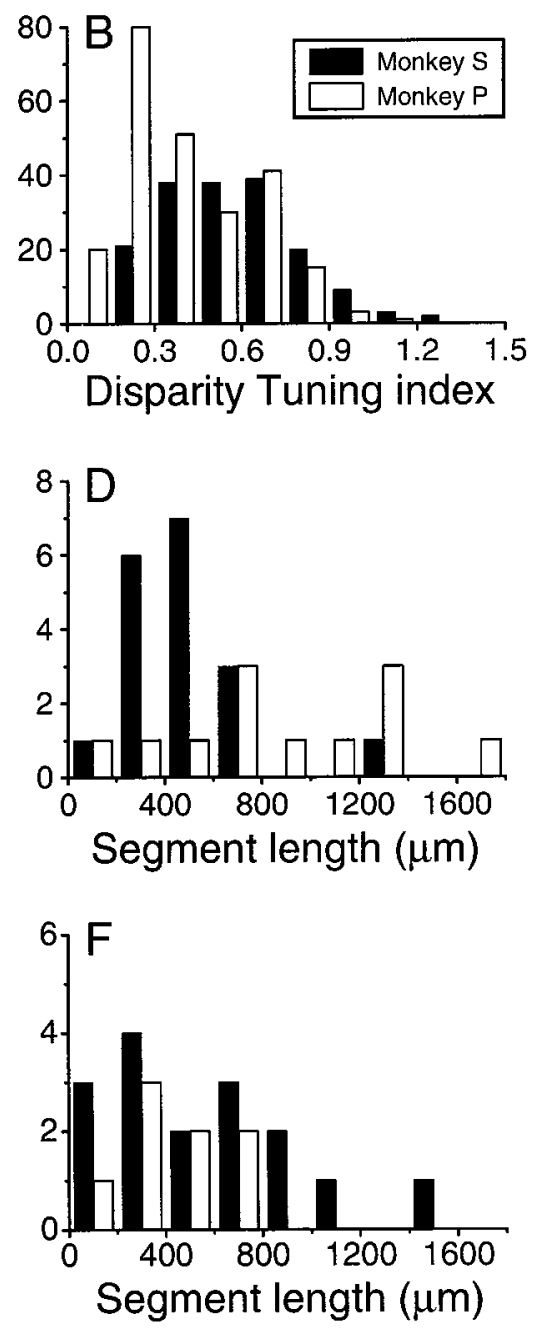

Figure 13. Analysis of the spatial organization of patches of weak and strong tuning for direction and disparity. $A$, Distribution of directiontuning indices for monkey $\mathrm{S}$ (filled bars) and monkey $\mathrm{P}$ (open bars). All data shown in this figure are from multiunit $(M U)$ recordings. $B$, Distribution of disparity-tuning indices. $C$, Distribution of lengths of penetration segments with poor direction tuning (direction-tuning index $<$ $0.5)$. Only segments from oblique penetrations are included in this data set. $D$, Distribution of segment lengths with poor disparity selectivity (disparity-tuning index $<0.5$ ). $E$, Histogram of segment lengths with strong direction tuning (direction-tuning index $>0.5$ ). $F$, Histogram of segment lengths with strong disparity tuning (disparity-tuning index $>0.5$ ). worth remembering that even within these patches of poor directional tuning, as assessed by MU recording, most single units are actually directional (Fig. 5C). Within patches that are poorly tuned for disparity, however, most single units are also poorly tuned (Fig. 5A).

\section{Correlation between disparity selectivity and other response parameters}

To gain further insight into the organization of MT, we looked for relationships between disparity-tuning parameters (disparitytuning index and preferred disparity) and other response metrics. Specifically, we wanted to determine whether disparity selectivity could be predicted to some degree from other response parameters of MT neurons. To screen for potential relationships, we initially correlated each disparity-tuning parameter with seven other response metrics: RF size, RF eccentricity, preferred speed, direction-tuning index, preferred direction, percent surround inhibition, and ocular imbalance index (a measure of ocular dominance; see Materials and Methods). Because some of these parameters were not normally distributed, we used a nonparametric measure of correlation (Spearman's rank correlation). Parameters that were significantly correlated with either disparity-tuning index or preferred disparity were then subjected to a further multiple regression analysis to rule out effects of intervening variables and intersubject differences.

Disparity-tuning index was found to be significantly correlated with only two other variables: preferred speed and OII. Figure $14 A$ depicts the relationship between disparity-tuning index and preferred speed. The two variables are negatively correlated (Spearman $r=-0.48 ; p \ll 0.001 ; n=411$ ); the full range of disparity indices is observed at sites preferring low speeds $\left(<5^{\circ}\right)$ $\mathrm{sec}$ ), but robust disparity tuning is rarely observed at sites preferring high speeds. Thus regions of MT that are concerned with fast speeds may not contribute appreciably to stereopsis. It is worth contrasting this result with the recent finding that stereoacuity in humans is maintained for velocities well above $100^{\circ} / \mathrm{sec}$, when low spatial frequency gratings are viewed (Morgan and Castet, 1995). If the findings of Morgan and Castet apply equally well to stimuli with broad spatial frequency tuning (like our random-dot patterns), then MT neurons may not be able to support depth perception at very high velocities.

Figure $14 B$ shows the relationship between disparity-tuning index and OII. Small values of OII indicate that responses measured through the two eyes were nearly equal in magnitude; large values indicate that one eye was dominant. These two variables 


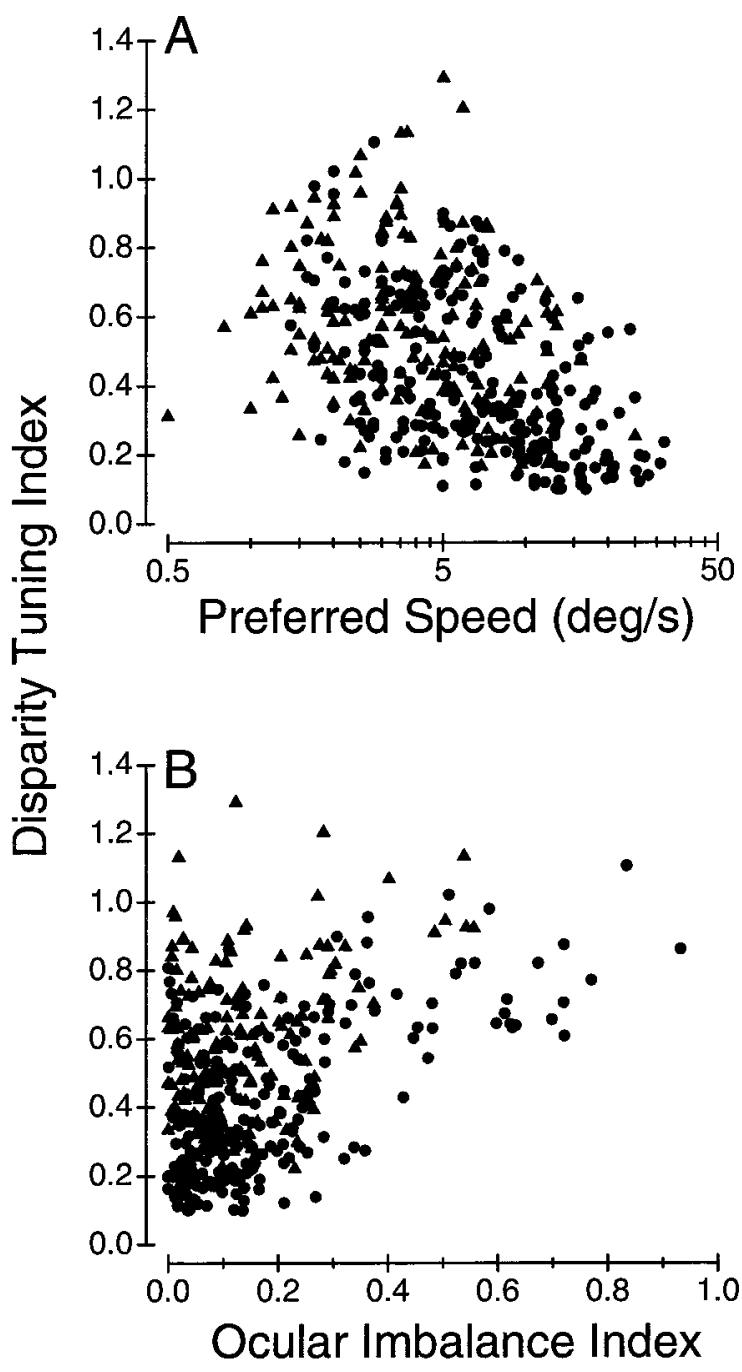

Figure 14. Correlations of disparity-tuning index with other response parameters. $A$, Relationship between disparity-tuning index and preferred speed of motion. All data are derived from MU responses. Circles represent data from monkey P; triangles indicate data from monkey S. Note that preferred speed is plotted on a logarithmic axis. $B$, Relationship between disparity-tuning index and ocular imbalance index (OII, see Materials and Methods). OII is a measure of ocular dominance; small values denote matched response levels for the two eyes; large values indicate that one eye is dominant.

are positively correlated (Spearman $r=0.33 ; p \ll 0.001 ; n=$ 411); the full range of disparity tuning is observed for small OII, but only strong disparity tuning is observed for large OII. In agreement with previous reports (Maunsell and Van Essen, 1983b; Felleman and Kaas, 1984), we find that most responses in MT are ocularly balanced, but substantial ocular imbalance, when it occurs, is a good predictor of strong disparity selectivity.

To eliminate the possibility that these correlations are driven by covariation with other parameters or by monkey differences, we performed a multiple regression analysis with disparity-tuning index as the dependent variable and with speed, eccentricity, and OII as independent variables. All three independent variables were found to have an approximately log-normal distribution; we therefore log transformed each variable for this analysis. We also added a dummy variable to account for different intercepts for the two monkeys, as well as three interaction terms (speed $\times$ monkey, eccentricity $\times$ monkey, and OII $\times$ monkey) to allow different slopes for the two monkeys. The relationship between disparity tuning and (log) speed survived the multiple regression analysis (partial $r=-0.18 ; p=0.0004$ ), as did the relationship between disparity tuning and ( $\log )$ OII (partial $r=0.15 ; p=0.002)$. The partial correlation for (log) eccentricity was suggestive but not significant (partial $r=0.07 ; p=0.13$ ). The effect of monkey identity was weakly significant $(p=0.036)$, reflecting a small difference in the mean disparity-tuning index between the two monkeys (see also Fig. $13 B$ ). The eccentricity $\times$ monkey interaction was marginally significant $(p=0.041)$ as well, whereas the other interaction terms were not $(p>0.2)$. From this analysis, we conclude that the correlations shown in Figure 14 are robust.

Among the parameters that were not correlated with the disparity-tuning index, two are worth noting. First, the disparitytuning index is not related to the direction-tuning index (Spearman $r=-0.02 ; p=0.64 ; n=411$ ), indicating that regions of strong (or weak) direction tuning do not coincide with regions of strong (or weak) disparity tuning. Second, the disparity-tuning index is not correlated with percent surround inhibition (Spear$\operatorname{man} r=0.05 ; p=0.58 ; n=318$ ). Thus, patches of strong or weak disparity tuning in macaque MT do not seem to be correlated with the patches of strong or weak surround inhibition ("interbands" and "bands," respectively) found by Born and Tootell (1992) in owl monkey MT.

We also performed this same set of analyses using the absolute value of preferred disparity (abbreviated here as APD) as the dependent variable. Our initial screening suggested that APD was modestly, but significantly, correlated with three independent variables: preferred speed, eccentricity, and percent surround inhibition. However, none of these relationships survived a multiple regression analysis that included all three independent variables along with various terms (as described above) to account for interanimal differences ( $p>0.25$ for all partial correlations). Thus, any relationships that might exist between APD and other independent parameters are too weak to be detected reliably in our data set. It is worth noting that APD and preferred direction of motion were completely unrelated (Spearman $r=0.05 ; p=$ $0.36 ; n=319)$.

\section{Rates of change of disparity and direction preferences}

The correlation analyses described above revealed no evidence of a systematic relationship between the preferred values of direction and disparity or between the tuning indices for direction and disparity. Thus, these data suggest that the maps for direction and disparity in MT are primarily independent. To explore this issue further, we examined rates of change of direction and disparity, as well as the interaction between these two. Overall, the absolute rates of change of the two parameters were unrelated. As shown below, however, an interesting relationship emerged between the sign of change for direction and disparity.

We computed rates of change by fitting straight lines to selected segments of plots of preferred disparity versus penetration distance, as illustrated in Figure 15 (see also Albright et al., 1984). We fitted straight lines, using linear regression, to all possible (overlapping) windows of $400 \mu \mathrm{m}$ length, which corresponds to five data points. Filled squares and triangles in Figure $15 A$ show the data points comprising two such windows, and the solid and dashed straight lines, respectively, show the best-fitting lines. The dashed line (Fig. 15A) clearly provides an acceptable fit to the triangles, whereas the solid line is a poor fit to the squares. We quantified the goodness-of-fit by computing a normalized root- 


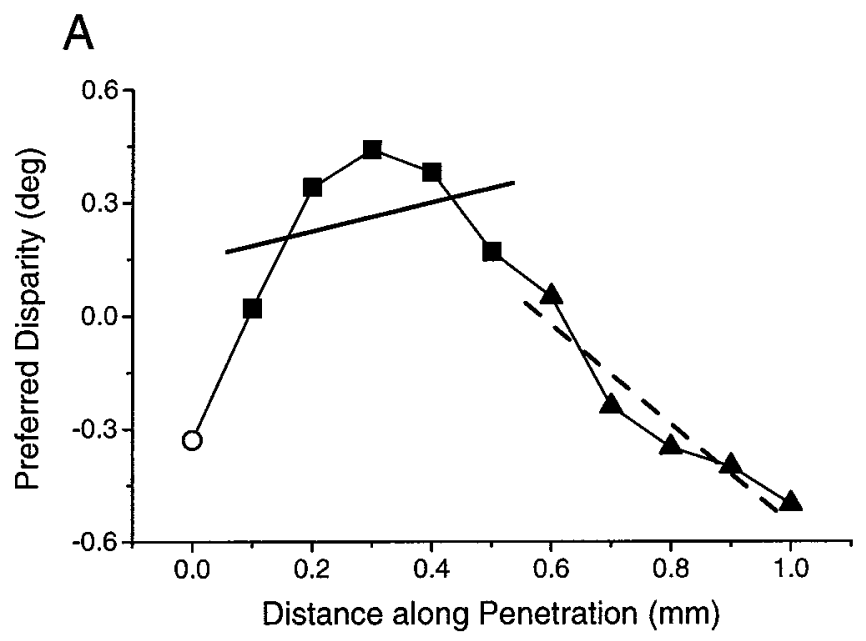

B

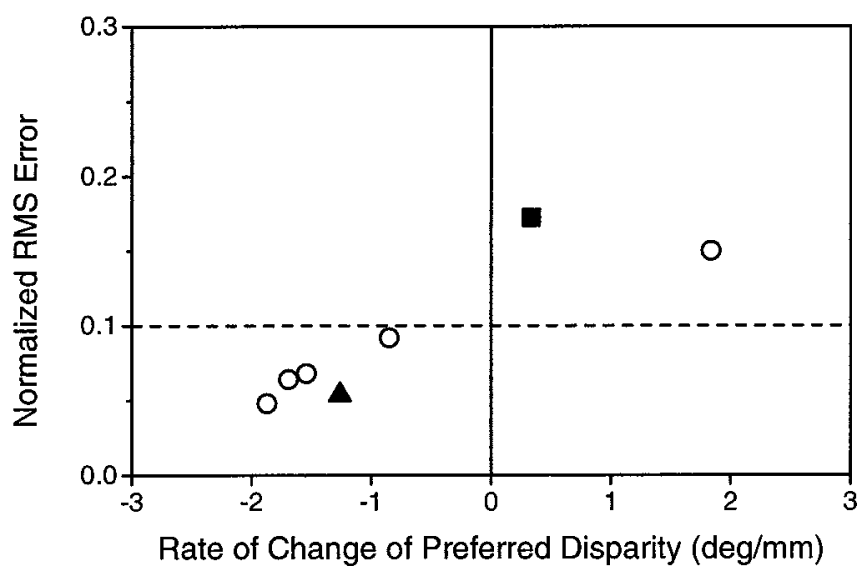

Figure 15. Method for computing rates of change of direction and disparity preference. $A$, Variation of preferred disparity with distance along an oblique penetration from monkey S. To measure slopes, we used a $400 \mu \mathrm{m}$ sliding window (five data points) and performed linear regression on the data in this window. The window was then moved by one data point, and the process was repeated. Filled squares and triangles indicate two possible positions of the sliding window, and the solid and dashed straight lines show the best linear fits to data in these two windows. The open circle denotes a data point not included in either window. $B$, Normalized root-mean-square $(R M S)$ error of the linear fit plotted as a function of the slope of the best-fitting line. The dashed horizontal line indicates an $R M S$ error level of 0.1 , which was our cut-off for accepting the linear fits. In this penetration, linear fits were accepted for five of seven positions of the sliding window, including the position denoted by the filled triangle. Two fits were rejected, including the one indicated by the filled square.

mean-square (RMS) error (the RMS error divided by the absolute mean value of the five data points), and we accepted fits for which this metric was $<0.1$. Figure $15 B$ shows the RMS error plotted against the slope of the best-fitting line for all possible 400 $\mu \mathrm{m}$ windows applied to this penetration. The filled triangle and square in Figure $15 B$ correspond to the segments illustrated in Figure $15 A$, whereas open circles in Figure $15 B$ denote other segments. Two of the data points in Figure $15 B$ (including the filled square) lie above our criterion (dashed line) RMS error of 0.1 ; slopes from these fits were excluded from further analyses. Importantly, this procedure is both objective and quantitative, precluding any subjective bias in the selection of segments to be fit.

This slope analysis was performed on all penetrations for both the preferred disparity and the preferred direction of motion. Figure 16 shows the pooled results using a $400 \mu \mathrm{m}$ analysis window. We chose this window because it is approximately the size of a directional "hypercolumn" in MT (Albright et al., 1984); however, we obtained very similar results using 300 and $500 \mu \mathrm{m}$ windows as well. Figure $16 A$ shows absolute rates of change of preferred disparity measured along oblique penetrations. The median values are $0.96 \% \mathrm{~mm}$ for monkey S (Fig. 16 A, open bars) and $2.25 \% \mathrm{~mm}$ for monkey $\mathrm{P}$ ( filled bars), and this difference is highly significant (Mann-Whitney $U$ test, $Z=5.43$; $p \ll 0.001$ ). The larger rates of change in monkey $\mathrm{P}$ could result from an overall difference in the angle of oblique penetrations between the two animals, but we will argue against this possibility below. Rather, this difference likely results from the fact that monkey $\mathrm{P}$ exhibited a larger range of absolute preferred disparities $\left(0-2.5^{\circ}\right.$; median $\left.=0.65^{\circ} ; n=187\right)$ than monkey $\mathrm{S}\left(0-1.3^{\circ} ;\right.$ median $=0.30^{\circ}$; $n=149)$. If the total range of disparities is organized similarly in the two animals, we would expect to observe larger rates of change in monkey $\mathrm{P}$.

Figure $16 B$ shows rates of change of preferred disparity measured from normal penetrations in monkey $P$. The median value of $0.39 \% / \mathrm{mm}$ is substantially smaller than the medians from oblique penetrations in either monkey (Mann-Whitney $U$ test, $Z=4.52 ; p \ll 0.001$ compared with monkey $\mathrm{S} ; Z=6.73 ; p \ll$ 0.001 compared with monkey $\mathrm{P})$. This difference provides further support for the notion that preferred disparity is organized in vertical columns in MT.

Figure $16 C$ shows the distribution of absolute rates of change of preferred direction for oblique penetrations. Median values are 169.1 and $166.1^{\circ} / \mathrm{mm}$ for the two monkeys. The fact that these values are not significantly different (Mann-Whitney $U$ test, $Z=$ $0.19 ; p=0.85$ ) suggests that the angles of oblique penetrations are actually quite similar in the two monkeys. Note also that our largest rates of change for preferred direction, in the range from 300 to $500^{\circ} / \mathrm{mm}$, agree well with the maximum value reported by Albright et al. (1984), which was $420^{\circ} / \mathrm{mm}$. Lastly, Figure $16 \mathrm{D}$ shows direction data from normal penetrations. Here also, the median value of $60.1 \% \mathrm{~mm}$ for normal penetrations is significantly lower than the median values for oblique penetrations (MannWhitney $U$ test, $Z=4.75 ; p \ll 0.001$ compared with monkey $\mathrm{S}$; $Z=4.89 ; p \ll 0.001$ compared with monkey $\mathrm{P}$ ), consistent with the known columnar organization for preferred direction in MT.

Comparing rates of change for direction and disparity on a site-by-site basis can provide a sensitive assay of the relationship between the maps for these two variables. For this analysis we selected a subset of the data from Figure 16 for which rates of change were available for both preferred direction and disparity in each $400 \mu \mathrm{m}$ window. Some of the data segments could not be used because either preferred direction or disparity could not be defined at some points within a given window (because of poor tuning).

We first compared absolute rates of change for direction and disparity and found no correlation between these variables (linear regression, $r=-0.09 ; p>0.3 ; n=89$ ). Thus, for example, one variable does not change rapidly while the other changes slowly. However, we did observe an interesting relationship between signed rates of change for direction and disparity, as shown in Figure 17. Surprisingly, regression analysis revealed a modest, but significant, negative correlation between these variables $(r=$ 
Figure 16. Summary of rates of change of preferred direction and disparity measured from oblique and normal penetrations. In each histogram, data from monkey S are indicated by open bars, and data from monkey $\mathrm{P}$ are denoted by filled bars. Note that these distributions give absolute values of the rates of change. $A$, Rates of change of preferred disparity in oblique penetrations. $B$, Rates of change of preferred disparity in normal penetrations. $C$, Rates of change of preferred direction in oblique penetrations. $D$, Rates of change of preferred direction in normal penetrations.

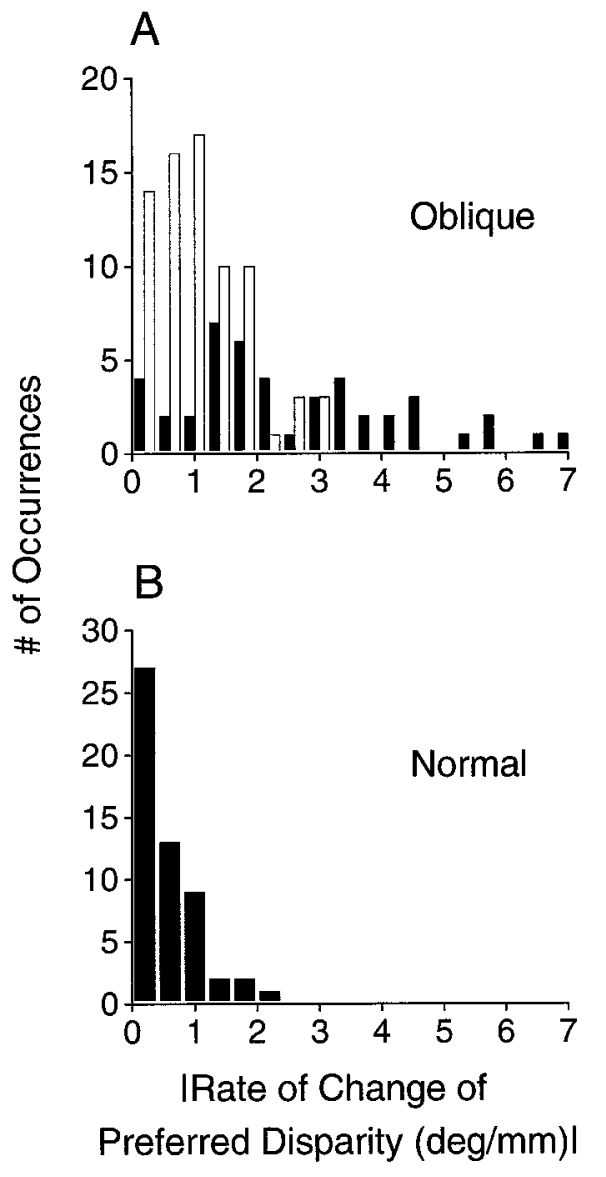

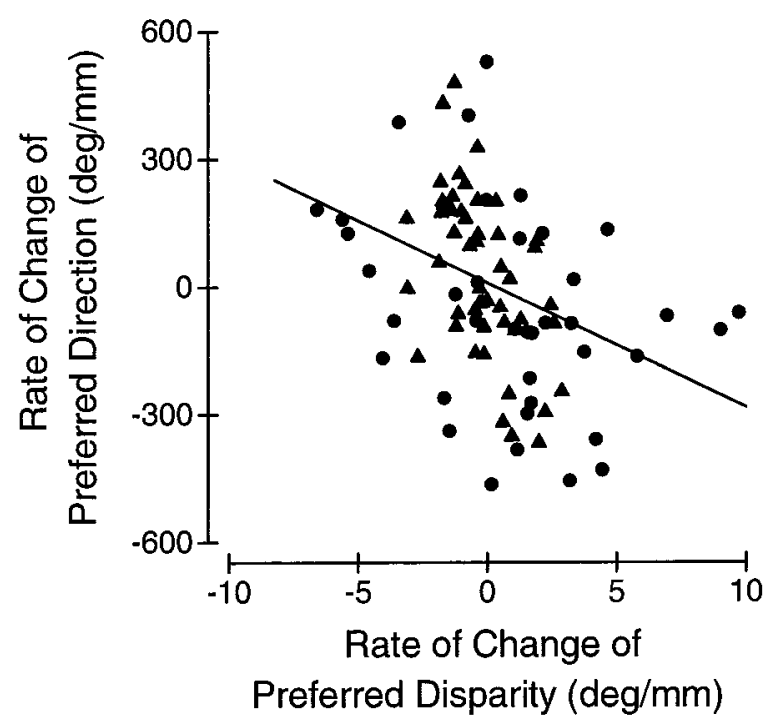

Figure 17. Comparison of signed rates of change in preferred direction and disparity. Triangles and circles denote data from monkeys $\mathrm{S}$ and $\mathrm{P}$, respectively. The solid line is the best linear fit (linear regression). Positive values on the $y$-axis correspond to preferred direction rotating counterclockwise; positive values on the $x$-axis correspond to preferred disparity changing from near to far.

$-0.35 ; p<0.001 ; n=89)$. Hence, when preferred direction changes in the counterclockwise direction (positive rate), preferred disparity tends to change from far to near (negative rate); when preferred direction rotates clockwise, preferred disparity tends to change from near to far. Although the functional rationale for this particular relationship is not clear to us, the data nevertheless suggest that the maps of direction and disparity are registered such that rates of change are inversely correlated.

\section{DISCUSSION}

We have demonstrated that MT contains a rather elaborate functional organization for binocular disparity. This organization, which is illustrated schematically in Figure 18, has two main features. First, the distribution of disparity-selective neurons within MT is nonuniform. Some patches of MT (Fig. 18, colored patches) are strongly selective for disparity, whereas other patches exhibit weak disparity tuning. Second, within the strongly tuned patches, the preferred disparity typically varies smoothly across the surface of MT (Fig. 18, color coding), with little variation in disparity preference within a vertical column.

Although Figure 18 represents our current conception of the organization of disparity in MT, we emphasize that some aspects of this figure are oversimplified or poorly constrained by the data. Boundaries between disparity-selective and nonselective regions are shown as sharp transitions in Figure 18. Although this was often the case, as in Figures $7 B$ and $9 B$ where these transitions occur within 100-150 $\mu \mathrm{m}$, other oblique penetrations exhibited a more gradual variation in disparity selectivity. Thus, the sharp boundaries of Figure 18 should not be taken literally. In addition, we know little about the actual shapes of the disparity-selective patches in MT and little about how consistently preferred disparity is organized within these patches. Finally, we know relatively little about how the maps of disparity and direction of motion are registered in MT. We did not observe any significant correlations 


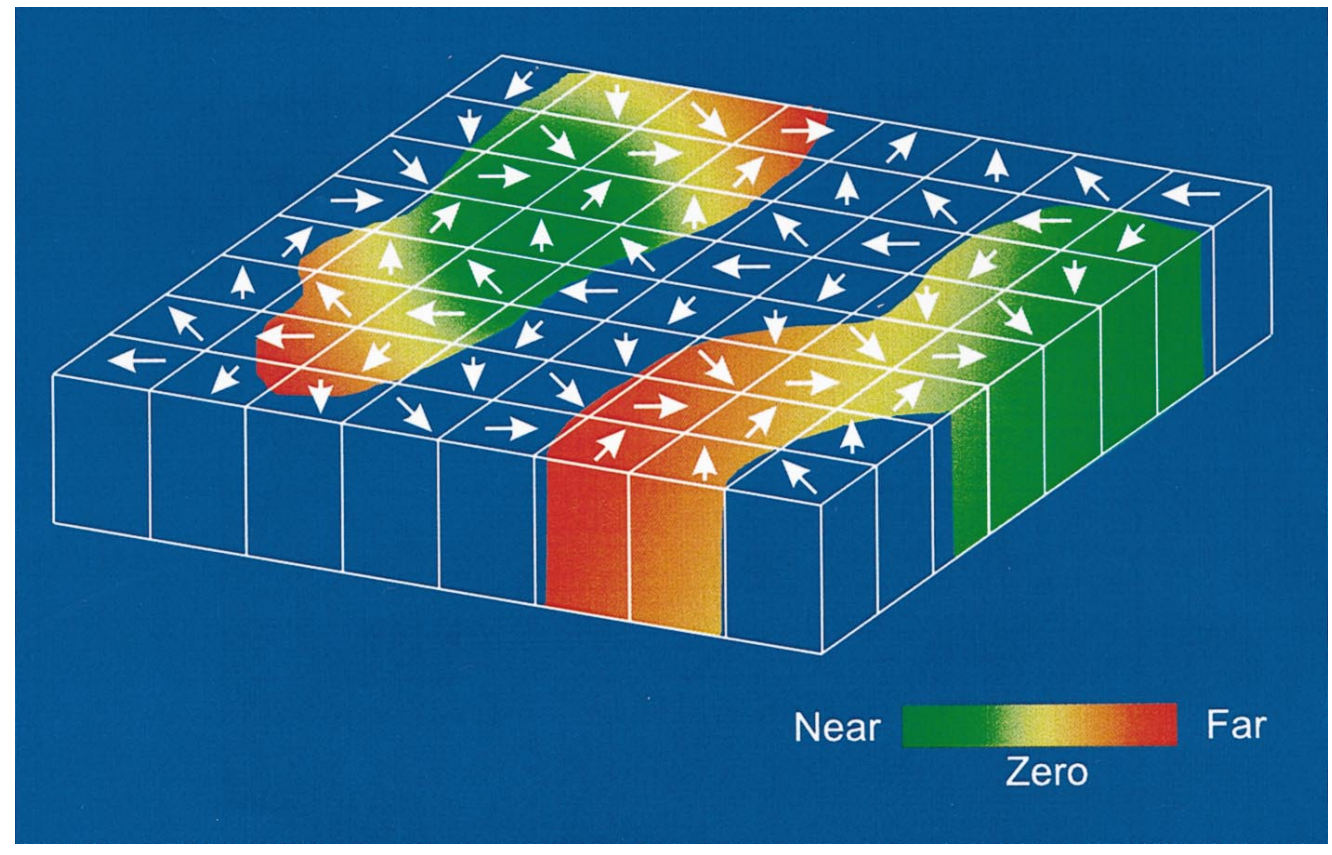

Figure 18. Schematic summary of the functional architecture of MT, with regard to binocular disparity and direction of motion. The top surface of this slab corresponds to the surface of MT, and the height of the slab corresponds to the thickness of the cortex. Arrows denote the preferred direction of motion of MT neurons in each direction column. Note that we have simply shown direction to vary smoothly across the surface of MT in both dimensions. We have not attempted to depict the direction map accurately, nor have we attempted to depict any discontinuities in the direction map (for comparison, see Malonek et al., 1994). Preferred disparity is colorcoded, with green representing near disparities, red representing far disparities, and yellow indicating zero disparity. Dark blue regions denote portions of MT that have poor disparity tuning. between preferred directions and disparities or between direction- and disparity-tuning indices. We did observe a weak inverse correlation between signed rates of change of direction and disparity (Fig. 17); however, this correlation is not intentionally represented in Figure 18. Many of the above issues are exceedingly difficult to resolve by making electrode penetrations through MT in an awake animal. Functional mapping studies, involving either optical imaging or 2-deoxyglucose autoradiography, could address these issues more incisively.

\section{Control of vergence eye movements}

When measuring disparity-tuning curves from an awake animal, one needs to be concerned with vergence eye movements. In control experiments, we have observed that monkeys will converge or diverge their eyes when a large random-dot pattern is presented over the fixation point at a nonzero disparity. Presumably, the animals are converging their eyes toward the depth plane of the dots. Our practice of presenting test patterns eccentrically and having a zero-disparity background surrounding the fixation point (Fig. 1) is generally effective at discouraging this behavior. Nevertheless, if the monkeys were to track even partially the disparity of our stimulus with their vergence posture, we might measure little or no disparity tuning when, in fact, the neurons were strongly selective for disparity.

To ensure that vergence eye movements did not contaminate our results, we implanted eye coils binocularly into one animal and measured vergence posture during experiments in which disparity-tuning curves were collected. If the monkey verged his eyes on the depth plane of the dots in the receptive field, there should be a strong correlation (with near unity slope) between the vergence angle and horizontal disparity (both expressed in degrees of visual angle). In contrast, data pooled across 33 experiments from monkey $\mathrm{S}$ reveal no such correlation at all (linear regression, $r=0.02$; slope $=0.003 ; p>0.5 ; n=305)$. Among the individual experiments, moreover, there was a significant correlation $(p<0.05)$ in only one case. Thus, we conclude that our methods of stimulus presentation were effective at minimizing vergence tracking, even though we did not behaviorally enforce a vergence requirement.

\section{Discrete classes of disparity tuning?}

In their pioneering work on disparity selectivity in awake monkeys, Poggio and Fischer (1977) defined three basic classes of disparity tuning. Tuned-excitatory (and -inhibitory) neurons had sharp disparity-tuning curves that were symmetric about the peak, and these neurons were tuned to disparities near zero. Near and far neurons had asymmetric tuning curves and preferred a broad range of disparities on either side of zero. More recently, Poggio et al. (1988) have expanded the tuned-excitatory group to include tuned-near, tuned-zero, and tuned-far types.

Many studies of disparity selectivity have classified neurons into the three basic types of Poggio and Fischer (1977). Indeed, many of the neurons in our sample from MT (e.g., Fig. 4) are also well described by these basic types. However, other researchers have questioned whether all neurons fall cleanly into one of these discrete categories (LeVay and Voigt, 1988). Our data from MT also question the discreteness of these categories. For example, Figures $3 B$ and 10 show disparity-tuning curves that are intermediate between the tuned-near and tuned-inhibitory types of Poggio et al. (1988). Moreover, the notion of discrete classes of disparity tuning seems incompatible with the idea of a continuous map of preferred disparities. Consider the data of Figures 6 and 7, for example. Within the disparity-selective portion of this oblique penetration, preferred disparities progressed smoothly from near to far and back to near (Fig. 7B), with no suggestion of discrete steps. Thus, our data support the idea that disparitytuning curves exhibit a continuum of shapes and preferences.

It is also worth noting that many of our tuning curves that peak at a nonzero disparity tend to be asymmetric about this peak (e.g., Figs. 4B, $C, 6,10$ ). This behavior is consistent with models in which disparity tuning arises via phase shifts between RF subunits that are periodic in space (see, e.g., DeAngelis et al., 1995; Ohzawa et al., 1997). Other tuning curves, however, are quite symmetric around a nonzero disparity (e.g., Fig. 4A, open 
circles); these data may result from similar, but spatially disparate, RFs in the two eyes.

\section{Other evidence of disparity architecture}

Blakemore (1970) initially suggested that "constant depth" columns are present in striate cortex of the anesthetized cat, and he showed that preferred disparity could remain quite constant along normal penetrations. Blakemore did not record along tangential penetrations, however, so he could not demonstrate any systematic progression of preferred disparities across the surface of V1. In a more recent quantitative study of cat striate cortex, LeVay and Voigt (1988) demonstrated a modest, but statistically significant, tendency for preferred disparities to be clustered along tangential penetrations. However, this tendency was much weaker than what we have observed in MT [compare LeVay and Voigt (1988), their Fig. 10C, with our Fig. 12A, filled circles]. We are not aware of similar published data for macaque V1, but this comparison suggests that functional architecture for disparity may be substantially more pronounced in MT than in V1.

In macaque V2, there is evidence that disparity-selective neurons are found in discrete patches that correlate with the thick cytochrome oxidase stripes (Hubel and Livingstone, 1987; Peterhans and von der Heydt, 1993; Roe and Ts'o, 1995). Because MT receives direct ascending input from the thick stripes in V2 (DeYoe and Van Essen, 1985; Shipp and Zeki, 1985, 1989), it is tempting to speculate that these V2 afferents terminate selectively in the disparity-tuned patches of MT and avoid the poorly tuned patches. There is no direct evidence to support this conjecture, but it merits investigation.

With regard to disparity preference, previous studies have reported that neurons having similar disparity tuning are clustered within V2 (Hubel and Wiesel, 1970; Clarke et al., 1976; Hubel and Livingstone, 1987; Ts'o et al., 1990). Unfortunately, disparity-tuning curves were not measured quantitatively in some of these studies, and they were all performed with anesthetized, paralyzed animals, making the determination of retinal correspondence (zero disparity) quite difficult. In addition, quantitative analyses were not performed to eliminate the possibility that the observed sequences of disparity preference arose by chance. Nevertheless, the preponderance of evidence does support the notion that disparity has a systematic organization in V2. Thus, the organization for disparity that we observe in MT may be at least partly inherited, rather than generated de novo. Further studies will be necessary to resolve these questions.

\section{Role of MT in visual processing}

A wealth of data suggests that MT is intimately involved in motion perception (for review, see Albright, 1993). MT neurons are highly direction selective (Zeki, 1974), are organized in direction columns (Albright et al., 1984), are extremely sensitive to weak motion signals (Britten et al., 1992), and can be electrically stimulated to alter perceptual judgments of direction of motion (Salzman et al., 1992; Salzman and Newsome, 1994). Lesions of MT also produce profound deficits in motion discrimination (Newsome and Pare, 1988; Pasternak and Merigan, 1994; Orban et al., 1995). These and other results have led to the view that MT is highly specialized for processing motion information.

Recent studies, however, point to an important role for MT in depth perception. The present study indicates that MT neurons are organized in disparity columns. In addition, we have recently exploited this columnar organization for disparity to microstimulate clusters of MT neurons with similar disparity tuning. Stimu- lation of MT produces strong perceptual biases in a depth discrimination task, and these biases are predictable from the disparity tuning of the stimulated neurons (DeAngelis et al., 1998). Moreover, stimulation of MT biases depth judgments even when there is no motion in the visual stimulus, suggesting that the disparity signals in MT contribute to depth perception for stationary as well as for moving objects. Other recent studies suggest that MT may be involved in computing depth from motion signals. The responses of MT neurons have recently been found to correlate with perceptual reports in an ambiguous structure-frommotion task (Chang et al., 1996; Dodd et al., 1997; Bradley et al., 1998). In addition, MT neurons have been shown to respond selectively to speed gradients across space, which may be used to compute surface orientation in depth (Xiao et al., 1997). Thus, MT seems to play a broader role in visual perception than was conceived previously. Because recent studies demonstrate that motion and disparity interact meaningfully in MT (Bradley et al., 1995), a promising avenue for future investigation concerns the possibility that motion parallax and disparity signals in MT are assembled to provide an integrated representation of depth in the visual world.

\section{REFERENCES}

Albright TD (1984) Direction and orientation selectivity of neurons in visual area MT of the macaque. J Neurophysiol 52:1106-1130.

Albright TD (1993) Cortical processing of visual motion. Rev Oculomotor Res 5:177-201.

Albright TD, Desimone R (1987) Local precision of visuotopic organization in the middle temporal area (MT) of the macaque. Exp Brain Res 65:582-592.

Albright TD, Desimone R, Gross CG (1984) Columnar organization of directionally selective cells in visual area MT of the macaque. J Neurophysiol 51:16-31.

Barlow HB, Blakemore C, Pettigrew JD (1967) The neural mechanism of binocular depth discrimination. J Physiol (Lond) 193:327-342.

Blakemore C (1970) The representation of three-dimensional visual space in the cat's striate cortex. J Physiol (Lond) 209:155-178.

Born RT, Tootell RB (1992) Segregation of global and local motion processing in primate middle temporal visual area. Nature 357:497-499.

Bradley DC, Qian N, Andersen RA (1995) Integration of motion and stereopsis in middle temporal cortical area of macaques. Nature 373:609-611.

Bradley DC, Chang GC, Andersen RA (1998) Encoding of threedimensional structure-from-motion by primate area MT neurons. Nature 392:714-717.

Britten KH, Shadlen MN, Newsome WT, Movshon JA (1992) The analysis of visual motion: a comparison of neuronal and psychophysical performance. J Neurosci 12:4745-4765.

Burkhalter A, Van Essen DC (1986) Processing of color, form, and disparity information in visual areas VP and V2 of ventral extrastriate cortex in the macaque monkey. J Neurosci 6:2327-2351.

Chang GC, Bradley DC, Andersen RA (1996) Neural correlate of 3-D structure from motion (SFM) perception in area MT. Soc Neurosci Abstr 22:1618.

Clarke PG, Donaldson IM, Whitteridge D (1976) Binocular visual mechanisms in cortical areas I and II of the sheep. J Physiol (Lond) 256:509-526.

DeAngelis GC, Freeman RD, Ohzawa I (1994) Length and width tuning of neurons in the cats primary visual cortex. J Neurophysiol 71:347-374.

DeAngelis GC, Ohzawa I, Freeman RD (1995) Neuronal mechanisms underlying stereopsis: how do simple cells in the visual cortex encode binocular disparity? Perception 24:3-31.

DeAngelis GC, Cumming BG, Newsome WT (1998) Cortical area MT and the perception of stereoscopic depth. Nature 394:677-680.

DeYoe EA, Van Essen DC (1985) Segregation of efferent connections and receptive field properties in visual area V2 of the macaque. Nature 317:58-61.

Dodd JV, Cumming BC, Newsome WT, Parker AJ (1997) Firing of V5 (MT) neurons reliably covaries with reported 3-D configuration in a 
perceptually-ambiguous structure-from-motion task. Soc Neurosci Abstr 23:1125.

Felleman DJ, Kaas JH (1984) Receptive-field properties of neurons in middle temporal visual area (MT) of owl monkeys. J Neurophysiol 52:488-513.

Felleman DJ, Van Essen DC (1987) Receptive field properties of neurons in area V3 of macaque monkey extrastriate cortex. J Neurophysiol 57:889-920.

Fujita I, Tanaka K, Ito M, Cheng K (1992) Columns for visual features of objects in monkey inferotemporal cortex. Nature 360:343-346.

Hubel DH, Livingstone MS (1987) Segregation of form, color, and stereopsis in primate area 18 . J Neurosci 7:3378-3415.

Hubel DH, Wiesel TN (1970) Stereoscopic vision in macaque monkey. Cells sensitive to binocular depth in area 18 of the macaque monkey cortex. Nature 225:41-42.

Hubel DH, Wiesel TN (1977) Ferrier lecture. Functional architecture of macaque monkey visual cortex. Proc R Soc Lond [Biol] 198:1-59.

Judge SJ, Richmond BJ, Chu FC (1980) Implantation of magnetic search coils for measurement of eye position: an improved method. Vision Res 20:535-538.

LeVay S, Voigt T (1988) Ocular dominance and disparity coding in cat visual cortex. Vis Neurosci 1:395-414.

Maldonado PE, Gödecke I, Gray CM, Bonhoeffer T (1997) Orientation selectivity in pinwheel centers in cat striate cortex. Science 276:1551-1555.

Malonek D, Tootell RB, Grinvald A (1994) Optical imaging reveals the functional architecture of neurons processing shape and motion in owl monkey area MT. Proc R Soc Lond [Biol] 258:109-119.

Maunsell JH, Van Essen DC (1983a) Functional properties of neurons in middle temporal visual area of the macaque monkey. I. Selectivity for stimulus direction, speed, and orientation. J Neurophysiol 49:1127-1147.

Maunsell JH, Van Essen DC (1983b) Functional properties of neurons in middle temporal visual area of the macaque monkey. II. Binocular interactions and sensitivity to binocular disparity. J Neurophysiol 49:1148-1167.

Maunsell JH, Van Essen DC (1987) Topographic organization of the middle temporal visual area in the macaque monkey: representational biases and the relationship to callosal connections and myeloarchitectonic boundaries. J Comp Neurol 266:535-555.

Morgan MJ, Castet E (1995) Stereoscopic depth perception at high velocities. Nature 378:380-383.

Mountcastle V (1957) Modality and topographic properties of single neurons of cat's somatic sensory area. J Neurophysiol 20:408-438.

Mountcastle V (1997) The columnar organization of neocortex. Brain 120:701-722.

Newsome WT, Pare EB (1988) A selective impairment of motion perception following lesions of the middle temporal visual area (MT). J Neurosci 8:2201-2211.

Ohzawa I, DeAngelis GC, Freeman RD (1997) Encoding of binocular disparity by complex cells in the cat's visual cortex. J Neurophysiol 77:2879-2909.

Orban GA, Saunders RC, Vandenbussche E (1995) Lesions of the superior temporal cortical motion areas impair speed discrimination in the macaque monkey. Eur J Neurosci 7:2261-2276.

Pasternak T, Merigan WH (1994) Motion perception following lesions of the superior temporal sulcus in the monkey. Cereb Cortex 4:247-259.

Payne BR, Berman N, Murphy EH (1980) Organization of direction preferences in cat visual cortex. Brain Res 211:445-450.
Peterhans E, von der Heydt R (1993) Functional organization of area V2 in the alert macaque. Eur J Neurosci 5:509-524.

Pettigrew JD, Nikara T, Bishop PO (1968) Binocular interaction on single units in cat striate cortex: simultaneous stimulation by single moving slit with receptive fields in correspondence. Exp Brain Res 6:391-410.

Poggio GF, Fischer B (1977) Binocular interaction and depth sensitivity in striate and prestriate cortex of behaving rhesus monkey. J Neurophysiol 40:1392-1405.

Poggio GF, Gonzalez F, Krause F (1988) Stereoscopic mechanisms in monkey visual cortex: binocular correlation and disparity selectivity. J Neurosci 8:4531-4550.

Robinson DA (1963) A method of measuring eye movement using a scleral search coil in a magnetic field. IEEE Trans Biomed Eng 10:137-145.

Roe AW, Ts'o D (1995) Visual topography in primate V2: multiple representation across functional stripes. J Neurosci 15:3689-3715.

Roy JP, Komatsu H, Wurtz RH (1992) Disparity sensitivity of neurons in monkey extrastriate area MST. J Neurosci 12:2478-2492.

Salzman CD, Newsome WT (1994) Neural mechanisms for forming a perceptual decision. Science 264:231-237.

Salzman CD, Murasugi CM, Britten KH, Newsome WT (1992) Microstimulation in visual area MT: effects on direction discrimination performance. J Neurosci 12:2331-2355.

Shipp S, Zeki S (1985) Segregation of pathways leading from area V2 to areas V4 and V5 of macaque monkey visual cortex. Nature 315:322-325.

Shipp S, Zeki S (1989) The organization of connections between areas V5 and V2 in macaque monkey visual cortex. Eur J Neurosci 1:333-354.

Shmuel A, Grinvald A (1996) Functional organization for direction of motion and its relationship to orientation maps in cat area 18 . J Neurosci 16:6945-6964.

Shoham D, Hübener M, Schulze S, Grinvald A, Bonhoeffer T (1997) Spatio-temporal frequency domains and their relation to cytochrome oxidase staining in cat visual cortex. Nature 385:529-533.

Tolhurst DJ, Thompson ID (1982) Organization of neurones preferring similar spatial frequencies in cat striate cortex. Exp Brain Res 48:217-227.

Tootell RB, Silverman MS, De Valois RL (1981) Spatial frequency columns in primary visual cortex. Science 214:813-815.

Ts'o DY, Gilbert CD, Wiesel TN (1990) Functional architecture of color and disparity in visual area 2 of macaque monkey. Soc Neurosci Abstr 16:293.

Weliky M, Bosking WH, Fitzpatrick D (1996) A systematic map of direction preference in primary visual cortex. Nature 379:725-728.

Xiao DK, Marcar VL, Raiguel SE, Orban GA (1997) Selectivity of macaque MT/V5 neurons for surface orientation in depth specified by motion. Eur J Neurosci 9:956-964.

Zeki SM (1973) Colour coding in rhesus monkey prestriate cortex. Brain Res 53:422-427.

Zeki SM (1974) Functional organization of a visual area in the posterior bank of the superior temporal sulcus of the rhesus monkey. J Physiol (Lond) 236:549-573.

Zeki SM (1978a) Functional specialisation in the visual cortex of the rhesus monkey. Nature 274:423-428.

Zeki SM (1978b) Uniformity and diversity of structure and function in rhesus monkey prestriate visual cortex. J Physiol (Lond) 277:273-290. 